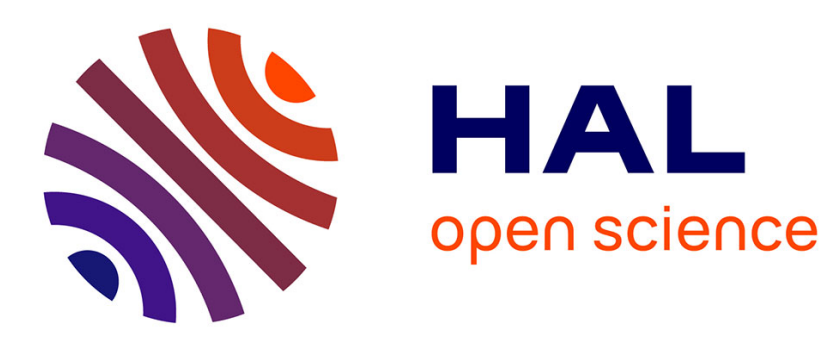

\title{
A Lattice-Boltzmann study of premixed flames thermo-acoustic instabilities
}

\author{
Karthik Bhairapurada, Bruno Denet, Pierre Boivin
}

\section{To cite this version:}

Karthik Bhairapurada, Bruno Denet, Pierre Boivin. A Lattice-Boltzmann study of premixed flames thermo-acoustic instabilities. Combustion and Flame, 2022, 240, pp.112049. hal-03582162

\section{HAL Id: hal-03582162 \\ https://hal.science/hal-03582162}

Submitted on 21 Feb 2022

HAL is a multi-disciplinary open access archive for the deposit and dissemination of scientific research documents, whether they are published or not. The documents may come from teaching and research institutions in France or abroad, or from public or private research centers.
L'archive ouverte pluridisciplinaire HAL, est destinée au dépôt et à la diffusion de documents scientifiques de niveau recherche, publiés ou non, émanant des établissements d'enseignement et de recherche français ou étrangers, des laboratoires publics ou privés. 


\title{
A Lattice-Boltzmann study of premixed flames thermo-acoustic instabilities
}

\author{
Karthik Bhairapurada $^{\mathrm{a}}$, Bruno Denet ${ }^{\mathrm{b}}$, Pierre Boivin $^{1 \mathrm{a}}$ \\ ${ }^{a}$ Aix Marseille Univ, CNRS, Centrale Marseille, M2P2, Marseille, France \\ ${ }^{b}$ Aix Marseille Univ, CNRS, Centrale Marseille, IRPHE, Marseille, France
}

\begin{abstract}
We present possibly for the first time Lattice-Boltzmann numerical simulations of thermo-acoustic instabilities of premixed flames. We study flames interacting with an imposed acoustic field where flames submitted to a parametric instability can be observed, as well as plane flames re-stabilized by the acoustic forcing. Self-induced thermo-acoustic oscillations of flames propagating in narrow channels are also studied, indicating an unexpected dependency with the channel width. For both excited and self-excited flames, results confirm that Lattice-Boltzmann method can capture the complex coupling between flame dynamics and acoustics.
\end{abstract}

Keywords: Lattice-Boltzmann methods; thermo-acoustic flame instabilities; premixed flames

\section{Introduction}

Lattice-Boltzmann (LB) methods [1] have become a mature technology in the field of isothermal external aerodynamics and aeroacoustics [2]. Their

\footnotetext{
${ }^{1}$ Corresponding author: pierre.boivin@univ-amu.fr 
base algorithm is the so-called Stream $\&$ collide procedure [3], in which all convective terms are accounted for by the Stream step, consisting of effortlessly moving data around on a Cartesian mesh (e.g. without the need of any interpolation). After the Stream step, the time is frozen via a splitting technique and a Collision step ensues, accounting for the non-linearity of the stress tensor only as a relaxation. The particular structure of the Stream \& collide algorithm, which can be seen as a Cartesian based solver in which only nearest-neighbors are required, forms the base of the success of LB methods [3], granting excellent dissipation properties [4] at an affordable cost [5].

Lifting the isothermal hypothesis and extending LB methods to compressible and reactive flows is non-trivial and a long-standing issue. One of the first models for reactive flows was presented by Succi et al [6] almost 25 years ago. Several contributions by Yamamoto and coworkers [7-10] ensued. Yet, no clear consensus has emerged as to the best way to simulate singlecomponent compressible flows using LBM, still an active topic of investigation among the community (see, e.g. [11-14] for selected studies published in the past year alone). In parallel of recent studies carried out in our group for compressible flows [15-17], we have developed a model to tackle reactive flows, and subsequently validated it on canonical combustion applications $[18,19]$ and a turbulent bluff-body flame [20]. The method was further validated following a combustion DNS benchmark [21], indicating that the CPU cost of a compressible reactive flow simulation is comparable or less than that of classical Navier-Stokes solvers in the low-Mach number approximation [22]. Note that another class LB methods is available for compressible flows - usually referred to as Discrete Boltzmann Methods - with increased 
focus on the thermodynamic non-equilibrium behaviors [23, 24]. They have been applied successfully to multiphase [25], multicomponent flows [26], as well as detonations $[27,28]$.

Given (i) the success of LB methods in the field of aeroacoustics [29, 30] and (ii) its competitiveness for combustion [22], the question of its applicability to thermo-acoustic instabilities (see a recent review for laminar premixed flames [31]) naturally arises and shall be the center of this contribution.

In the present paper, we will apply the LB method to the problem of interaction with acoustics of premixed flames propagating in tubes (or in our case in 2D channels), which has been a classical problem since the work of Markstein [32] (see also [33, 34]). Without acoustics, a flame propagating in a tube is submitted to the hydrodynamic Darrieus-Landau (DL) instability, leading to a cellular front for flames propagating downward. We have recently studied this instability with an LB method, and we were able to recover both linear and non-linear premixed flame propagation regimes, by comparing with experiments in Hele-Shaw cells [35]. With acoustics the problem is even more challenging for the hybrid LB solver: there is a two-way coupling between acoustics and the shape of the flame, and as the flame propagates, acoustics develops, typically in the middle of the tube. A simpler configuration is to study the forcing of the flame by an imposed acoustic field, usually obtained with a loudspeaker [36], but recently a vibroacoustic coupling has also been used $[37,38]$. In this paper, we will study both problems: a flame forced by a given acoustic field and the amplification of acoustics during the flame propagation in a $2 \mathrm{D}$ narrow channel $[39,40]$.

This paper consists of three main Sections. After a brief reminder of 
the LB hybrid model proposed [16, 20, 22, 35] and of the behavior of freely propagating unstable flames in slip and non-slip channels, we will study how acoustic forcing influences premixed flame propagation, and in particular its intrinsic DL instability. A large section is then dedicated to study selfinduced thermo-acoustic instabilities in narrow channels, providing new insights on the instability growth rate dependence on channel width.

\section{Lattice-Boltzmann model}

Numerical simulations are carried out with the PROLB software, which was recently adapted to tackle combustion problems [18-20, 22, 35]. The compressible core corresponds to the pressure-based LB model presented by Farag et al. for compressible flows [16]. It consists of an LB solver, accounting for mass and momentum conservation, coupled with a Finite Difference (FD) solver for energy and species equations.

In the LB solver, the probability density function $f_{i}$ of finding gas with velocity $\boldsymbol{c}_{\boldsymbol{i}}$ at position $\boldsymbol{x}$ is solved via a classical stream and collide algorithm, [3] following

$$
f_{i}(t+\Delta t, \boldsymbol{x})=f_{i}^{c o l}\left(t, \boldsymbol{x}-\boldsymbol{c}_{\boldsymbol{i}} \Delta t\right),
$$

for the streaming step, accounting for the convective part of Euler equations. In the streaming equation, $\boldsymbol{c}_{\boldsymbol{i}}$ corresponds to the $i^{\text {th }}$ discrete velocity of the D3Q19 lattice [3], and $(\Delta x, \Delta t)$ are the space and time discretisation. The collision then reads

$$
f_{i}^{c o l}(t, \boldsymbol{x})=f_{i}^{e q}(t, \boldsymbol{x})+\left(1-\frac{\Delta t}{\bar{\tau}}\right) f_{i}^{n e q}(t, \boldsymbol{x})+\frac{\Delta t}{2} F_{i}^{E}(t, \boldsymbol{x}),
$$

corresponding to the hybrid regularized collision model presented by Jacob et al. [41] relaxing $f_{i}$ towards the equilibrium population $f_{i}^{e q}$ at a relaxation 
time $\bar{\tau} . \quad \bar{\tau}$ is a function of the viscosity $(\mu)$, defined as $\bar{\tau}=\frac{\mu}{\rho c_{s}^{2}}+\frac{\Delta t}{2}$; here $c_{s}$ is the constant characteristic lattice velocity [3]. Full expressions for the equilibrium and non-equilibrium functions $\left(f_{i}^{e q}, f_{i}^{n e q}\right)$ and forcing term $F_{i}^{E}$ are provided in Appendix A for completeness, but the interested reader is referred to $[16,35]$ for their derivation.

Farag et al. [42] showed via Taylor expansion the equivalence of the above system with mass and momentum equations

$$
\begin{aligned}
\frac{\partial \rho}{\partial t}+\frac{\partial}{\partial x_{\alpha}}\left(\rho u_{\alpha}\right) & =0 \\
\frac{\partial \rho u_{\beta}}{\partial t}+\frac{\partial}{\partial x_{\alpha}}\left(\rho u_{\alpha} u_{\beta}\right) & =-\frac{\partial p}{\partial x_{\beta}}+\frac{\partial \mathcal{T}_{\alpha \beta}}{\partial x_{\alpha}},
\end{aligned}
$$

at second-order in time and space, where notations follow Poinsot \& Veynante combustion textbook [43]: $p$ is the thermodynamic pressure following a perfect multi-constituent gas law, and $\mathcal{T}_{\alpha \beta}$ is the viscous tensor.

Following our previous studies, energy and species equations are solved in a coupled way, under non-conservative form [19, 20, 22, 35]. The species equations read

$$
\rho \frac{\partial Y_{k}}{\partial t}+\rho u_{\alpha} \frac{\partial Y_{k}}{\partial x_{\alpha}}=\frac{\partial}{\partial x_{\alpha}}\left(-\rho V_{k, \alpha} Y_{k}\right)+\dot{\omega}_{k}
$$

where $Y_{k}$ is the mass fraction of the $k^{t h}$ species, $V_{k, \alpha}$ is the $\alpha$-component of its diffusion velocity, and $\dot{\omega}_{k}$ its reaction rate. Energy equation is solved in its sensible form as

$$
\rho \frac{\partial e}{\partial t}+\left(\rho u_{\alpha}\right) \frac{\partial e}{\partial x_{\alpha}}=-\frac{\partial q_{\alpha}}{\partial x_{\alpha}}+\left(\mathcal{T}_{\alpha \beta}-p \delta_{\alpha \beta}\right) \frac{\partial u_{\alpha}}{\partial x_{\beta}}
$$

where

$$
e=\sum_{k} Y_{k} e_{k}, \quad e_{k}=\int_{T_{0}}^{T} C_{v, k}(T) d T-\frac{R T_{0}}{W_{k}}+\Delta h_{f, k}^{0}
$$


obtained with NASA polynomials. $R=8.314 \mathrm{~J} \mathrm{~mol}^{-1} \mathrm{~K}^{-1}$ is the perfect gas constant, $T_{0}$ is the standard reference temperature and $W_{k}$ is the atomic weight of species $k$. Lastly, the heat flux $q_{\alpha}$ in Eq. (5) reads

$$
q_{\alpha}=-\lambda \frac{\partial T}{\partial x_{\alpha}}+\rho \sum_{k=1}^{N} h_{k} Y_{k} V_{k, \alpha}
$$

with $\lambda$ the thermal conductivity, obtained from the temperature-dependent viscosity $\mu$ assuming constant Prandtl number Pr:

$$
\lambda=\frac{\mu c_{p}}{\operatorname{Pr}} .
$$

Diffusion velocities are defined as in [35], with constant Schmidt numbers for each species and a correction velocity to enforce numerical mass conservation [43].

Note that the choice of treating the additional quantities (energy and species) as single scalars rather than additional distributions was investigated at length in previous studies (see, e.g. [16, 44, 45]) and was shown to preserve excellent dissipation properties, as well as the $2^{\text {nd }}$ order accuracy, for a very competitive cost [20, 22]. For instance, in [19], the cost per time step of including 9 species (transport properties and source terms included via a 12step hydrogen mechanism) and energy is less than four times that of classical athermal LBM.

Numerical parameters. All simulations hereafter are performed using a spatial discretization $\Delta x=\delta_{L} / 13$, where $\delta_{L}$ is the thermal flame thickness, based on maximum temperature gradient [43]. This was shown to be sufficient for an accurate resolution of the flame when single-step chemistry is used $[19,35]$. The discretization is also very close to the value $\Delta x \approx \delta_{L} / 15$ 
used by Jiménez et al [39]. The time-step is chosen setting 0.6 as maximum Courant-Friedrichs-Lewy number, defined as

$$
\mathrm{CFL}=\frac{\left|u_{\infty}\right|+c}{\Delta x / \Delta t}
$$

where $c=\sqrt{\gamma \cdot r \cdot T}$ is the sound speed, $\gamma$ is the heat capacity ratio and $r=R / W$, where $W$ is the mean molecular weight.

Thermo-chemical parameters. Throughout the paper, the gas consists of a multi-constituent mixture $\left(\mathrm{C}_{3} \mathrm{H}_{8}, \mathrm{O}_{2}, \mathrm{CO}_{2}, \mathrm{H}_{2} \mathrm{O}, \mathrm{N}_{2}\right)$, in which a one-step propane chemistry is considered:

$$
\mathrm{C}_{3} \mathrm{H}_{8}+5 \mathrm{O}_{2} \longrightarrow 3 \mathrm{CO}_{2}+4 \mathrm{H}_{2} \mathrm{O}
$$

The net progress rate of the reaction is expressed as

$$
\dot{Q}=A \cdot e^{-E_{a} / R T} \cdot C_{\mathrm{C}_{3} \mathrm{H}_{8}} \cdot C_{\mathrm{O}_{2}}
$$

where $C_{\mathrm{k}}$ is the molar concentration of species $\mathrm{k}, A$ is the pre-exponential factor and $E_{a}$ is the activation energy. A temperature power-law is assumed for the viscosity $\mu$ (see, e.g. p. 231 in [46])

$$
\mu=\mu_{0}\left(\frac{T}{T_{0}}\right)^{0.7},
$$

$\mu_{0}=1.782 \times 10^{-5} \mathrm{~kg} \mathrm{~m}^{-1} \mathrm{~s}^{-1}$ and $T_{0}=300 \mathrm{~K}$.

The composition of fresh gases is then set as to obtain a target expansion ratio. Finally, the Arrhenius parameters, Schmidt and Prandtl numbers chosen as to obtain targeted values for flame speed and flame thickness. 


\section{Freely propagating flames}

\subsection{Preliminary definitions}

Let us introduce the following quantities used throughout this study. As in [39], the consumption speed is defined as

$$
S_{c}=-\frac{1}{\rho_{0} Y_{F}^{0} D} \int_{0}^{W} \int_{0}^{L} \dot{\omega}_{F} d x d y,
$$

where $F$ denotes the fuel (propane) component. It is often normalized with $S_{l}$, the laminar flame speed. Subscripts 0 (e.g. $\left.\rho_{0}\right)$ denote the fresh gases properties, whereas $b$ denotes burnt gases properties.

Channel dimensions are normalized by the flame diffusive thickness $\delta_{T}$, defined as the ratio of thermal diffusivity in fresh gases to the laminar flame speed (see Tab. B.1 for the numerical values). With the parameters used here, $\delta_{L} \approx 6 \delta_{T}$.

For the two-dimensional flames studied here, the flame position is computed as an integral over the channel volume $V$

$$
x_{f}=\frac{1}{V} \int \frac{T-T_{0}}{T_{b}-T_{0}} d V
$$

It is oriented such as $x_{f}=0$ when the tube is filled with fresh gases and $x_{f}=1$ when filled with burnt gases.

\subsection{Darrieus-Landau instability}

Our starting point is our recent experimental/numerical investigation of the DL instability in the context of Hele-Shaw cell [35]. In this article, we successfully validated the growth rate and linear behavior of the DL instability, and in particular its gain dependence on wavelength, illustrated in 
Fig. 1. For wavelengths $\lambda$ greater than a cut-off value $\lambda_{c}$, positive growth rates are obtained, and the instability grows, whereas the instability damps when $\lambda<\lambda_{c}$. The non-linear regime was also investigated in a large HeleShaw cell, showing excellent agreement between experimental results, analytical results from the Michelson-Sivashinsky equation, and numerical results obtained with ProLB.

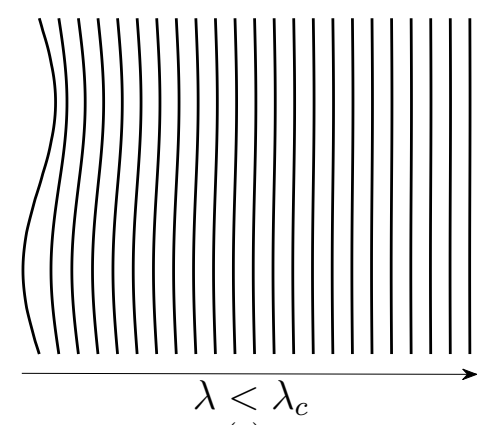

(a)

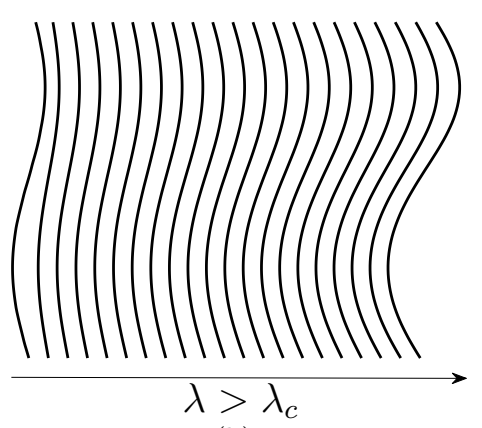

(b)

Figure 1: Observation of growth/decay of DL instability. (Flame profiles propagation is from left to right): ( $\boldsymbol{a})$ Instability decay at wavelength $\left(\lambda=40 \delta_{T}\right)$, below $\lambda_{c}$. (b) Instability growth at wavelength $\left(\lambda=43 \delta_{T}\right)$, above $\lambda_{c}$.

\subsection{Cut-off width in presence of walls}

To study the growth of a single wave perturbation in Fig. 1, a twodimensional periodic domain of width $\lambda$ was considered, with two open ends. In transitioning to channel flames, let us first study the impact of using the following boundary conditions on the cut-off wave length:

- periodic conditions, as in Fig. 1 and [35],

- slip adiabatic walls (or symmetry boundary condition), 
- non-slip adiabatic walls.

With the parameters detailed in Tab. B.1, simulations were carried out with the three aforementioned boundary conditions as to assess their impact on the DL cutoff wave length.

Figure 2 reports flame profiles propagation (from left to right), for each boundary condition, and for width chosen closely above and below the cutoff value. For width $W$ values below the cut-off wave length $\lambda_{c}$, the initial perturbation damps, and the flame becomes planar again.

In the last plot of Fig. 2e, the corresponding normalized consumption speeds are reported. Because of the change in flame surface, the front is significantly accelerated in all configurations $W>\lambda_{c}$, whereas it stays to values close to its laminar value $S_{l}$ when the instability damps $\left(W<\lambda_{c}\right)$.

By comparing the periodic and slip conditions results of Figs. 1 and 2, we can deduce that the cut-off wave length is approximately halved using slip conditions $\left(22.5<\lambda_{c} / \delta_{T}<25\right.$, vs $\left.40<\lambda_{c} / \delta_{T}<45\right)$. This result is rather intuitive: an adiabatic slip wall is equivalent to a symmetry condition, so the pattern observed with periodic conditions can be reproduced with half the width (and then mirrored to obtain the periodic case).

Imposing $u=0$ at the wall significantly modifies the flow-field by establishing a Poiseuille velocity profile. For this reason, the flame front does not remain exactly planar even for $W<\lambda_{c}$, and progresses slightly faster close to the wall (see Fig. 2c). The cut-off wavelength is also significantly decreased $\left(17.5<\lambda_{c} / \delta_{T}<19\right.$, vs $\left.22.5<\lambda_{c} / \delta_{T}<25\right)$.

Imposing non-slip conditions also leads to a more slanted flame above 
Slip

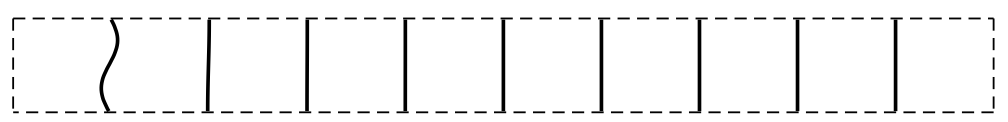

(a) $\mathrm{W}=22.5 \delta_{T}$

Slip

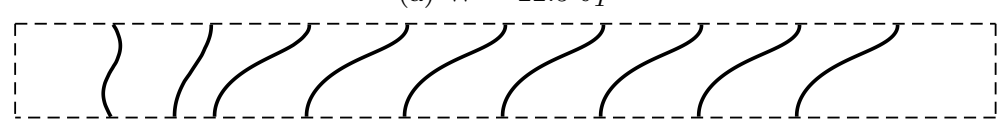

(b) $\mathrm{W}=25 \delta_{T}$

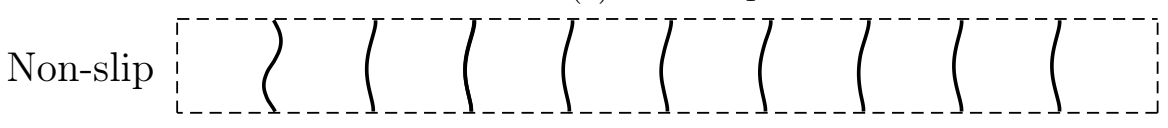

(c) $\mathrm{W}=17.5 \delta_{T}$

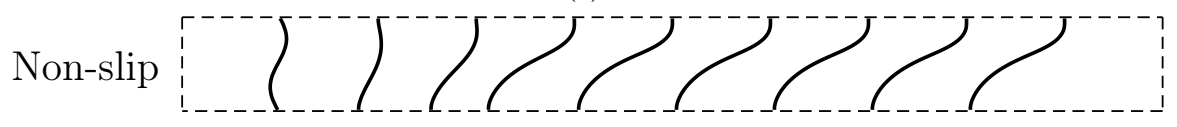

(d) $\mathrm{W}=19 \delta_{T}$

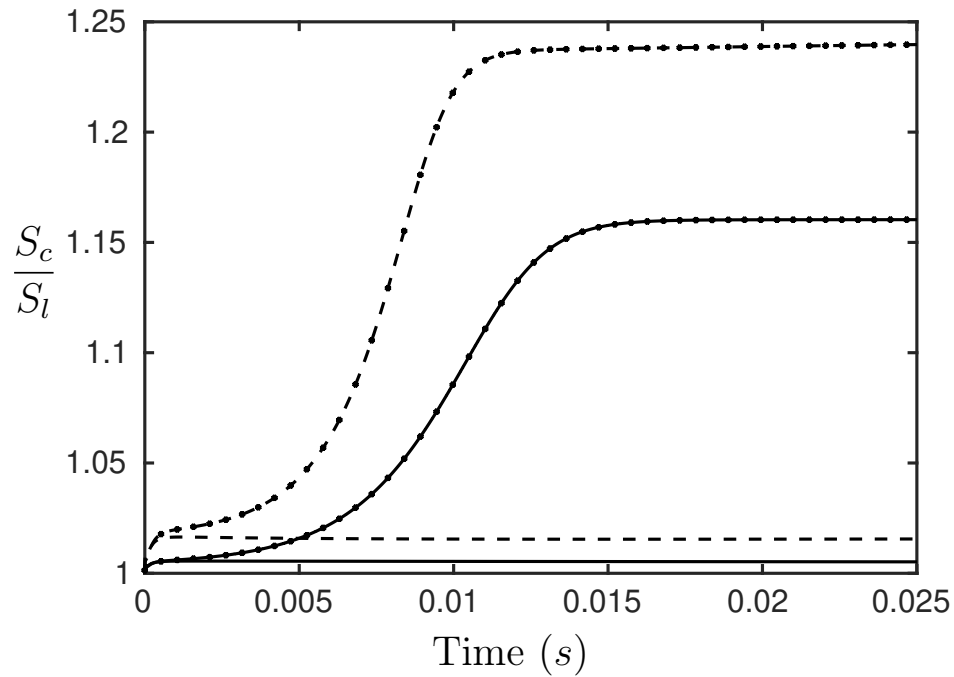

(e) The Flame consumption speed

Figure 2: Growth/Decay of DL instability in presence of walls. Flame shapes are depicted by the iso-contours of temperature $(1500 \mathrm{~K})$ (propagating left to right):

(a) DL instability decay between slip walls below cut-off wavelength $\left(W=22.5 \delta_{T}\right)$;

(b) DL instability growth between slip walls above cut-off wavelength $\left(W=25 \delta_{T}\right)$;

(c) DL instability decay between non-slip walls below cut-off wavelength $\left(W=17.5 \delta_{T}\right)$;

(d) DL instability growth between non-slip walls above cut-off wavelength $\left(W=19 \delta_{T}\right)$;

(e) The flame consumption speed in between slip and non-slip walls, depicted by solid and dash lines respectively. A marker on the line denotes $W>\lambda_{c}$. 
the cut-off width, leading to a significant increase in consumption speed.

\subsection{Narrow channel: symmetric vs asymmetric flames}

We have seen above that a perturbed flame turns to non-symmetric flame above a certain cut-off width, but if the initial flame profile is planar, the flame shape undergoes a change and becomes perfectly symmetric with the hump always pointing towards the burnt gases exit. It was found that the flame shape has two viable solutions [47] and it always chooses the nonsymmetric solution after some propagation time.

These two solutions are shown in Fig. 3. Non-slip, adiabatic walls on the top and bottom with acoustically non-reflecting boundaries on the left and right are considered. Though it can be clearly seen that the consumption speed and the flame curvature of non-symmetric flame in both cases is the same, this phenomenon is important because the acoustics response of symmetric and non-symmetric flame were found to be very different [39].

\section{Acoustically forced flames}

Before carrying on to study the self-excited thermo-acoustic instabilities in narrow channels, it is important to check that the flame responds properly to imposed acoustic perturbations.

Let us consider a single wave perturbed flame inside a 2D periodic domain, with a non-reflecting outlet. An acoustic forcing where only the $(u+c)$ mode is triggered is used to inject acoustic waves at the inlet. The imposed acoustic field is such that, the wavelength of these acoustic waves is much larger than the thermal flame thickness $\left(\delta_{L}\right)$ and due to the non-reflecting 


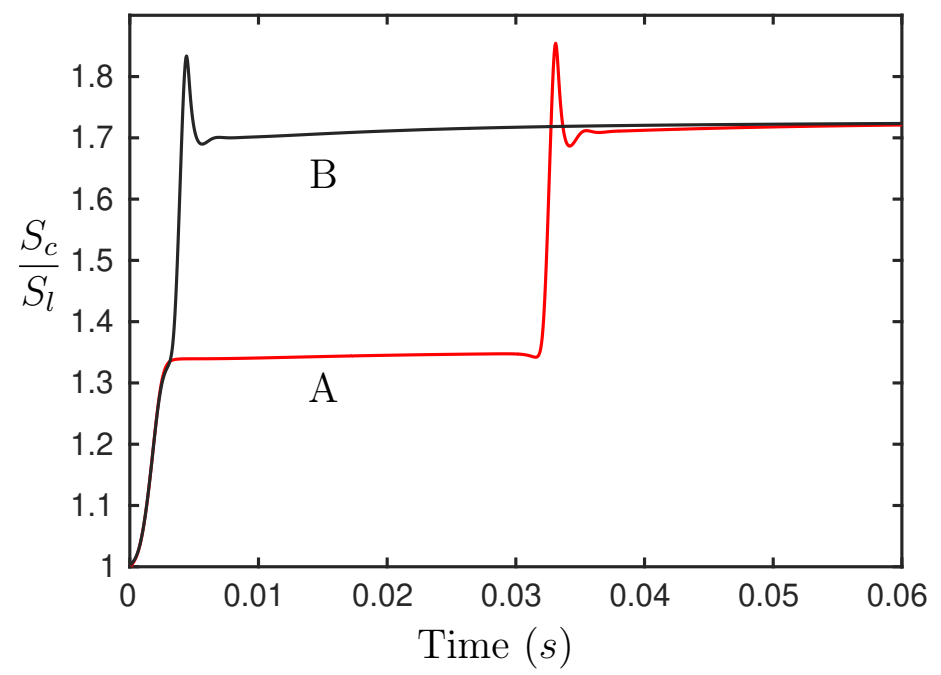

(a)

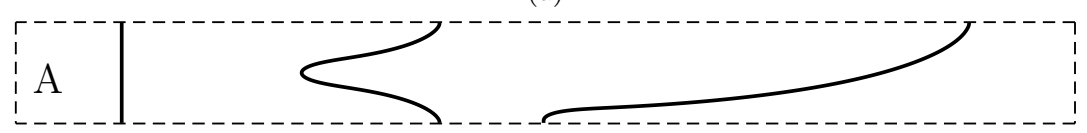

(b)

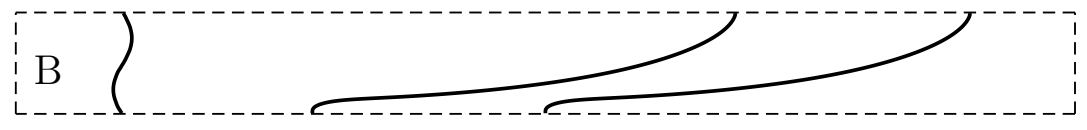

(c)

Figure 3: Illustration of flame shape transition in a channel of length $\left(L=1200 \delta_{T}\right)$ and width $\left(W=40 \delta_{T}\right)$ from initially planar $(\mathbf{A})$ and initially perturbed $(\mathbf{B})$ configurations: (a) Flame consumption speed for $(\mathbf{A})$ in red and (B) in black; (b) Flame shape transition from planar to symmetric and then non-symmetric; (c) Flame shape transition from perturbed to non-symmetric directly. 
conditions on the outlet they are not reflected back. The implementation details of the injection of the acoustic waves at the inlet are provided below:

$$
\begin{gathered}
u=u_{0}+u^{\prime}=u_{0}+\sqrt{\gamma r T_{0}} \epsilon \\
p=p_{0}+p^{\prime}=p_{0}(1+\gamma \epsilon) \\
\rho=\rho_{0}+\rho^{\prime}=\rho_{0}(1+\epsilon) \\
T=T_{0}+T^{\prime}=T_{0}(1+[\gamma-1] \epsilon)
\end{gathered}
$$

Here $\boldsymbol{x}_{\mathbf{0}}$ and $\boldsymbol{x}^{\prime}$ represent the mean and fluctuating quantities respectively and $\epsilon<<1$ is the fluctuation applied in the sinusoidal form as $\epsilon=A_{0} \sin 2 \pi f t$, where $A_{0}$ is the fluctuation amplitude and $f$ the frequency of the sinusoidal fluctuation. This formulation allows to selectively trigger the acoustic mode associated to the $(u+c)$ wave [48] on top of the mean flow $u_{0}$.

Markstein [32], depending on the amplitude of the reduced acoustic velocity $\left(\tilde{u}_{a}\right)$, noticed two distinct unstable regions: a region corresponding to a low acoustic excitation where the growth of the intrinsic instability (DL) is reduced and a higher acoustic excitation region also called a parametric instability region where the structures on the flame oscillate at half the acoustic frequency (twice the acoustic period). And in between these regions he observed a stable region where the flame becomes stable (planar) (see also [49] for the case of a conical flame).

We give here a short summary of the theory of parametric forcing of the flame by the acoustic field presented in [36] (see also [50, 51]). 
The effect of the acoustic forcing is, in this geometry, directly related to the characteristics of the hydrodynamic instability of the flame. It is well known that the equation for the growth rate of this instability is given by a quadratic equation $[35,52]$ :

$$
A(k) \sigma^{2}+B(k) \sigma+C(k)=0,
$$

where $\sigma$ is the growth rate of the perturbation with wavenumber $k . A(k)$, $B(k), C(k)$ are coefficients depending on gas expansion $E_{\rho}=\rho_{u} / \rho_{b}$, Markstein number $\mathcal{M}$, laminar flame speed $S_{l}$, flame thickness $\delta_{T}=D_{t h} / S_{l}$, Prandtl number $\operatorname{Pr}$ and Froude number $F r=S_{l}^{2} / g \delta_{T}(g$ is the acceleration due to gravity) as

$$
\begin{aligned}
& A(k)=\frac{E_{\rho}+1}{E_{\rho}}+\frac{E_{\rho}-1}{E_{\rho}} k \delta_{T}\left(\mathcal{M}-J \frac{E_{\rho}}{E_{\rho}-1}\right), \\
& B(k)=S_{l} k\left(2+2 E_{\rho} k \delta_{T}(\mathcal{M}-J)\right), \\
& C(k)=\frac{E_{\rho}-1}{E_{\rho}} \frac{k \delta_{T}}{F r}+S_{l}^{2} k^{2}\left(\left(E_{\rho}-1\right) \frac{k}{k_{c}}-\left(E_{\rho}-1\right)\left(1+\frac{1}{E_{\rho} F r}\left(\mathcal{M}-J \frac{E_{\rho}}{E_{\rho}-1}\right)\right)\right),
\end{aligned}
$$

where the cutoff wavenumber $k_{c}$ is given by $k_{c}^{-1}=\delta_{T}\left(E_{\rho}^{\beta}+\frac{3 E_{\rho}-1}{E_{\rho}-1} \mathcal{M}-\frac{2 E_{\rho}}{E_{\rho}-1} J+\right.$ $(2 P r-1) H)$ and the integrals $J$ and $H$ can be found in [52]. If we use the amplitude $\alpha$ of the Fourier mode with wavenumber $k$ we simply obtain an oscillator with damping:

$$
A(k) \alpha_{t t}+B(k) \alpha_{t}+C(k)=0
$$

where only the coefficient $C(k)$ depends on the Froude number. Now with 
an acoustic forcing, the mean position of the flame is periodically displaced by a planar sound wave. This leads to the front experiencing an effective dimensionless acceleration given by:

$$
\frac{g \delta_{T}}{S_{l}^{2}}-\tilde{\omega}_{a} \tilde{u}_{a} \cos \tilde{\omega}_{a} t,
$$

replacing the Froude number in $C(k)$ with this dimensionless acceleration, we have now a parametric oscillator with damping. Here $\tilde{\omega}_{a}$ and $\tilde{u}_{a}$ are the reduced, acoustic frequency and acoustic amplitude respectively. A simple change of variable [36] leads to a Mathieu equation [53], so that the different zones, DL zone for low forcing (lower zone in red in Fig. 4), parametric instability zone (upper zone in red in Fig. 4) and parametrically re-stabilized flat flame in between can be computed. We use here an implementation using Mathematica for the Mathieu functions, described in [54]. In the literature simulations [55] can be found where the gravity term is varied sinusoidally in time as in Eq. (22) to which our results can be compared, the boundary conditions that we use to have a constant acoustic amplitude were described previously.

In a case with a zero gravity term we can observe these regions in Fig. 4. The zero acoustic amplitude line bordering on the lower red region is the well known DL instability which at larger wavenumbers is stabilized by thermodiffusivity. The lower red region above it with small reduced acoustic amplitude is the region of primary instability and the top red region is the region of secondary/ parametric instability. And the light-colored region in the same figure represents the parameters at which the flame is planar. The wavenumber corresponding to the width of the domain $\tilde{k}$ and the multiples of $\tilde{k}$ are indicated with black dots in Fig. 4. The reduced acoustic amplitude is given 


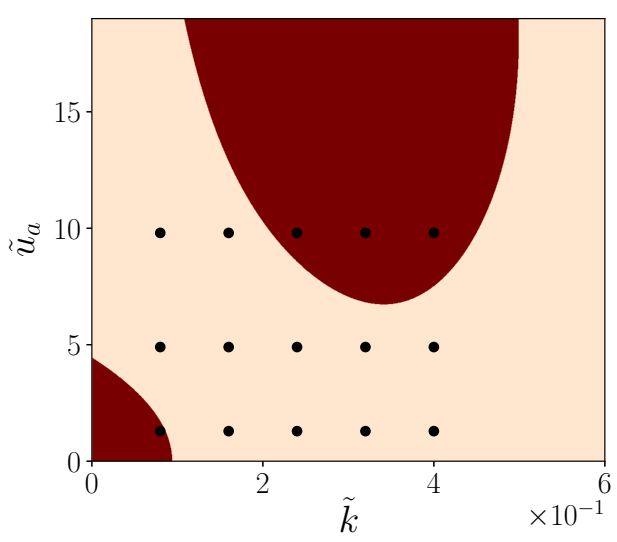

Figure 4: Stability diagram for parametrically excited flames with different regions based on the reduced acoustic amplitude and reduced wavenumber. The parameters are given in Tab. B.2

by $\left(\tilde{u}_{a}=u_{r m s} / S_{l}\right)$, where $u_{r m s}$ is the root mean square (rms) of the acoustic axial velocity measured at the flame position. The amplitude $\left(A_{0}\right)$ of the fluctuations $\epsilon$ in Eq.(15) - (18), corresponding to the three cases illustrated in the Fig. 4 are, $A_{0}=7.5 \times 10^{-4}$ for the lowest forcing applied, $A_{0}=2.5 \times 10^{-3}$ for the forcing that re-stabilizes the flame and $A_{0}=5.5 \times 10^{-3}$ for the high amplitude acoustic forcing, and the reduced acoustic frequency is $\tilde{\omega}_{a}=3.40$. For a given acoustic amplitude, the flame is unstable relative to one possible wavenumber if the black dot is inside one of the two red zones, DL zone at low amplitude or parametric zone at large acoustic amplitude. For a low acoustic amplitude we observe that only the wavenumber corresponding to the width of the domain $\tilde{k}$ is unstable: we have only one cell in the domain. For an intermediate amplitude no wavenumber is unstable: this is a parametrically flat flame. For a large acoustic amplitude, several wavenumbers are unstable (generally one is more unstable than the others), but not the lowest one, so we have a flame with several small cells, this is a parametrically unstable 
flame. All these behaviors are observed below in Fig. 5 .

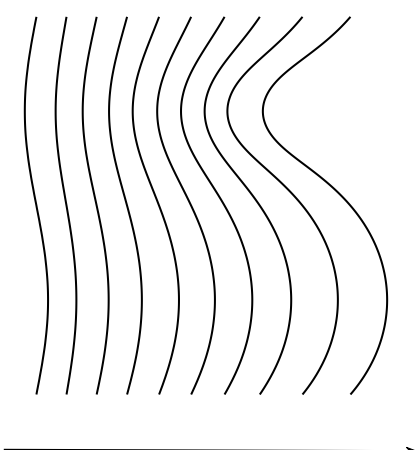

(a)

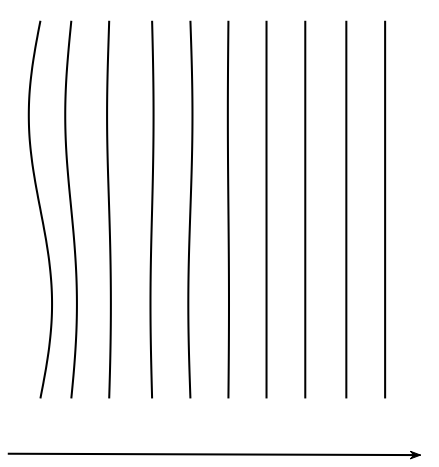

(c)

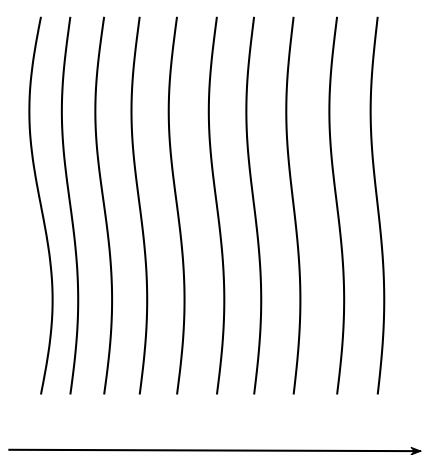

(b)

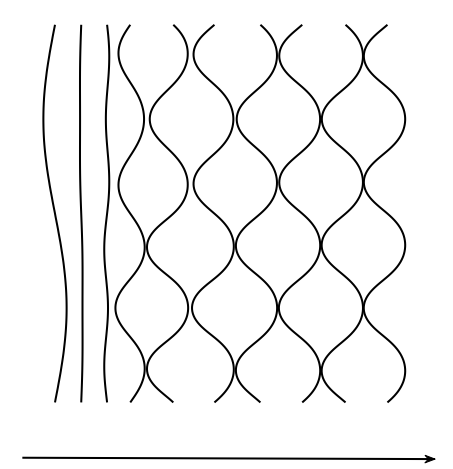

(d)

Figure 5: Illustration of flame behavior in different regions of Fig. 4. (iso-contours of temperature $1500 \mathrm{~K}$ are plotted from left to right at every forcing acoustic period $\left.10^{-3} \mathrm{~s}.\right)$ : $(\boldsymbol{a})$ Growth of DL instability without any acoustics; (b) Reduction DL instability amplitude by low amplitude acoustic forcing $\left(\tilde{u_{a}}=1.36\right) ;(c)$ A stable planar flame obtained by moderate amplitude acoustic forcing $\left(\tilde{u_{a}}=5.15\right) ;(d)$ Parametric instability due to high amplitude acoustic forcing $\left(\tilde{u_{a}}=10.23\right)$.

The parameters necessary to obtain the stability diagram are given in Tab. B.2. The chemistry along with the Schmidt numbers are also given. (refer Tab. B.3)

As mentioned earlier, for a chosen wavenumber $\tilde{k}<\tilde{k}_{c}$ (here $\tilde{k}=0.08$ ), 
the amplitude of the perturbation on the flame front grows in the absence of acoustics (see Fig. 5a); while the exact same initially perturbed flame front in the presence of imposed acoustics behaves differently. In practice, it was observed that the flame always chooses the most unstable wavenumber of the stability diagram.

On imposition of a low amplitude acoustics $\left(\tilde{u}_{a}=1.36\right)$ the amplitude of DL growth is reduced compared to the case without acoustics Fig. 5b.

Similarly when a moderate acoustic amplitude is imposed $\left(\tilde{u}_{a}=5.15\right)$, the flame becomes and remains planar after sometime, as depicted by Fig. 5c, because all corresponding wavenumbers are in the stable region.

And if the reduced amplitude is increased further $\left(\tilde{u_{a}}=10.23\right)$, the wavenumber of the flame front switches to the most unstable harmonic (in this case, the third one) as evidenced by Fig. 5d. There, iso-contours of temperature were plotted at each acoustic period to illustrate a fundamental characteristic of parametric instabilities: the flame front changes sign at every acoustic period.

\section{Self-excited flames in narrow channels}

Having validated the canonical answer of the flame front to acoustic perturbation, we may now study the thermo-acoustic instabilities, in which the acoustics are now directly triggered by the flame, sometimes leading to disastrous instabilities in the combustion chambers [31].

Hereafter, we consider thermo-acoustic instabilities in the narrow channel, depicted in Fig. 6. The flame is initialized with a 1D laminar flame profile centered on at $x_{f}=0.1$, the rest of the domain is filled with fresh gases. The 
flame front is initialized with a sine perturbation only when non-symmetric flames are considered. Atmospheric pressure is imposed at the outlet, making the boundary open to the exit of burnt gases but fully reflecting for the acoustics.

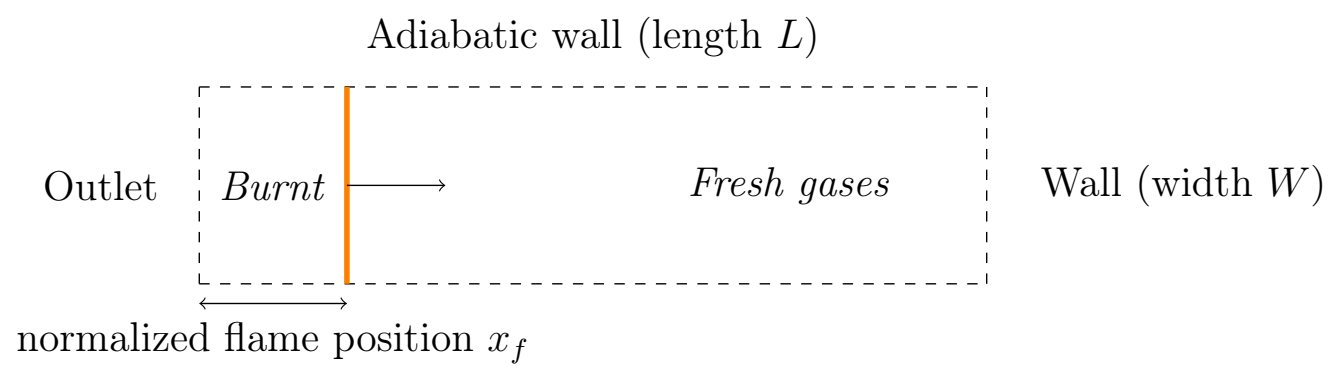

Figure 6: A sketch of the narrow channel under consideration.

Laminar flame speed $S_{l}$, flame diffusive thickness $\delta_{T}$ and the thermal gas expansion parameter $E_{T}$, have been chosen to obtain a growth rate $\sigma$ comparable with [39]. To obtain $\delta_{T}=10^{-4} \mathrm{~m}$, whilst preserving the flame speed $S_{l}=1 \mathrm{~m} \mathrm{~s}^{-1}$, a flame thickening model as proposed in [56] is used. Here a thickening factor of 4.7 is multiplied and divided to the diffusion terms and the source term respectively. Note that the acoustic Reynolds number defined as $R e_{a}=\delta_{T} c_{0} / \nu_{0}$ is now 2225 (almost 4.7 times larger than in [39]) and Lewis number is $L e=1$. And lastly the mixture composition is provided in Tab. B.3. Rest of the parameters used in the study of flame propagation in the narrow channels are provided in Tab. B.1.

For reference, a strict comparison between ProLB results and those from the study [39] using the same set of equations and parameters (single progress variable equation and constant molecular weight) is given in Appendix C, providing an additional validation for the present model. 
Through a number of numerical simulations, we shall successively (i) check that the generated instabilities correspond to the channel's natural eigen frequency, (ii) provide new insight on the growth rate dependence on width, and (iii) illustrate self-induced DL stabilization in the channel.

\subsection{Eigenmode analysis}

The first acoustic mode of a semi-open tube/channel of length $\mathrm{L}$ is the quarter-wave frequency

$$
f_{0}=\frac{c_{0}}{4 L},
$$

where $c_{0}$ is the gas sound speed. If a thin flame $\left(\delta_{T} \ll L\right)$ is present in the tube, one can approximate the first mode frequency $f$ [43] as the solution of

$$
\tan \left(\frac{2 \pi f L}{c_{0}}\left(1-x_{f}\right)\right) \tan \left(\frac{2 \pi f L}{c_{b}} x_{f}\right)=\Gamma,
$$

where

$$
\Gamma=\rho_{0} c_{0} / \rho_{b} c_{b}
$$

is the acoustic impedance ratio, and $x_{f}$ is the normalized flame position defined in Eq. (14). Note that to derive Eq. (24), an 1D problem is assumed, the flame is considered as a thin discontinuity separating fresh and burnt conditions at position $x_{f}$. Indeed, for $x_{f}=0$, corresponding to a tube filled of fresh gases, Eq. (24) leads to the simple quarter-wave expression corresponding to the fresh gases sound speed Eq. (23).

Let us now repeat the configuration presented in Fig. 3 using non-slip adiabatic walls ( $L=1200 \delta_{T}$ and $W=40 \delta_{T}$, above the DL cut-off width), and an asymmetric initial perturbation (case B). As stated earlier, a fixed 
pressure outlet $(p=1 \mathrm{~atm})$ is used to let the acoustic mode develop in the channel.

Evolution for consumption speed and pressure at the closed end is reported in Fig. 7.a. The mean consumption speed history indicates that the flame directly becomes asymmetric (as in case B of Fig. 3), but both the consumption speed and pressure at the wall now start oscillating, with maximum oscillations in the second half of the channel. Upon measuring the period of the oscillation as a function of flame position $x_{f}$, one can compare the period of the instability with the period corresponding to the main tube frequency Eq. (24). Fig. 7 compares the numerical period (black dots) with the analytical formula (solid line). Expectingly, the period decreases from its quarter-wave value obtained with the fresh gases sound speed $c_{0}$ to the value obtained using burnt gases sound speed $c_{b}$, when the channel gases are fully burnt. Fresh and burnt gases quarter-wave values Eq. (23) are denoted in the plot by the horizontal dashed lines. 

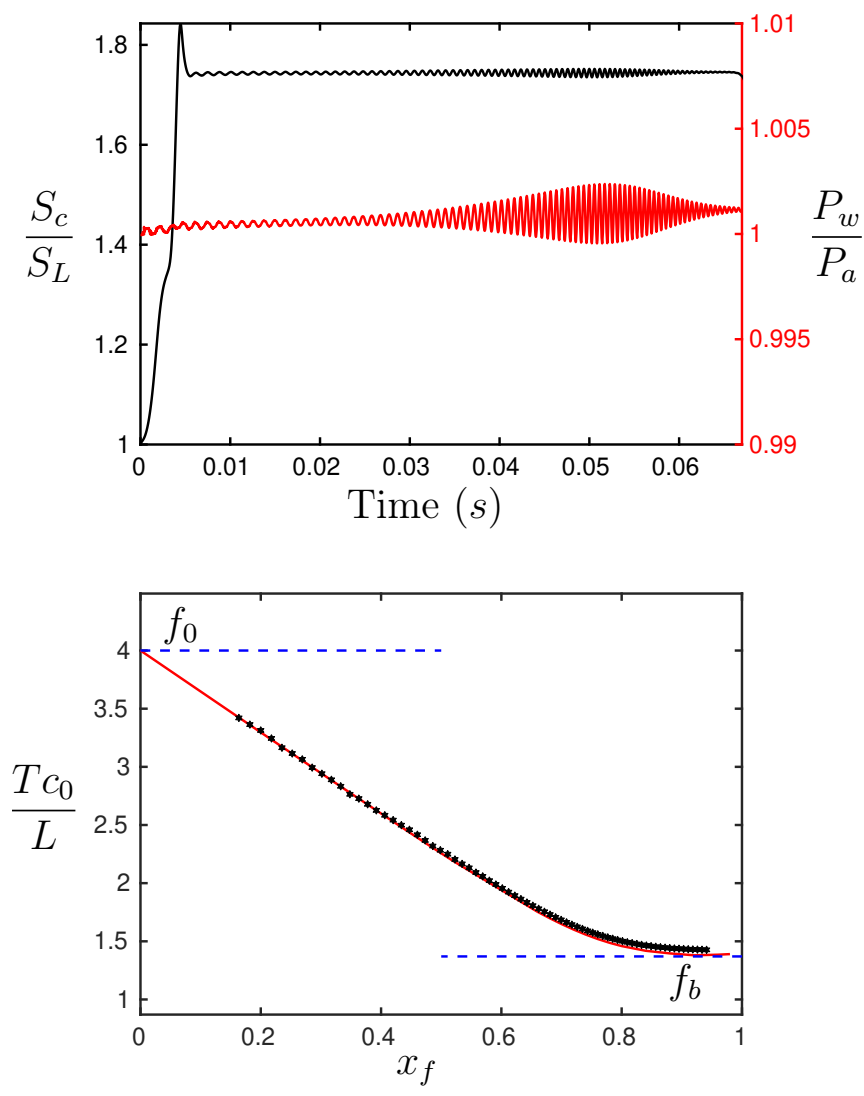

Figure 7: Thermo-acoustics inside a channel of length $\mathrm{L}=1200 \delta_{T}$ and width $\mathrm{W}=40$ $\delta_{T}$. (a) Flame consumption speed, $S_{c} / S_{l}$ (Black) and Pressure at the closed end, $P_{w} / P_{a}$ (Red). (b) Period of oscillations (T) for a flame propagating from $x_{f}=0$ to $x_{f}=1$ (Black); Acoustic eigen modes of the channel (red) from Eq. (24); top and bottom dashed blue lines represent the fresh $f_{0}$ and burnt $f_{b}$ quarter-wave values. 


\subsection{A study of the instability gain}

Having validated that the instability obtained in the previous Section corresponds indeed to the expected instability, related to the quarter-wave mode of the tube, let us now study the influence of the following parameters:

- flame shape (symmetric or asymmetric) and tube length,

- channel width.

Flame shape and tube length. The amplification of instabilities is a function of both the flame shape and the eigen frequency. By studying the amplitude gain of consumption speed oscillations in a similar configuration, Jiménez et al. [39] recently showed that symmetric flames are more unstable at higher frequencies, whereas non-symmetric flames respond more to lower frequencies.

Since the excitation frequency corresponds to the quarter-wave mode, this effect can readily be studied by varying the channel length. In the following, we consider channel length $L=800 \delta_{T}$ and $L=2400 \delta_{T}$, while keeping constant the width $W=40 \delta_{T}$. In both tubes, we run two computations, one initialized with a planar front (as in case A of Fig. 3) to let the symmetric flame develop, and a second one with an asymmetric initial front (case B of Fig. 3) to force early transition to asymmetric flame.

Figure 8 reports consumption speed histories in the four configurations. In the short tube (Fig. 8a), it is clear that the symmetric flame (curve A) is more prone to thermo-acoustic instability: the asymmetric flame does not show any instability in such a short tube. 
When the eigen frequency is divided by 3 for a channel 3 times longer (Fig. 8b), the opposite behavior is obtained: the asymmetric flame (B) is strongly unstable, whereas the symmetric flame (A) only shows damped oscillations. Note that once case A transitions to asymmetric flame, the instability grows again.

Influence of channel width. To the authors' knowledge, the question of the instability gain dependence on channel width is not yet reported in the literature: parametric studies on such configurations are oftentimes expensive. This is made easier with the present LB framework because of a significantly reduced cost: we reported in [22] that simulation costs of the compressible LB solver are comparable or lower than classical low-Mach reactive flows solvers (in which acoustic waves are not resolved).

In the following, a channel of length $L=1200 \delta_{T}$ is considered, and only asymmetric flames are considered (as in the case B of Fig. 3). Upon varying the channel width from $W=17.5 \delta_{T}$ (just below cutoff, see Fig. 2) up to $W=40 \delta_{T}$, we observed a non-monotonous dependence of the flame instability. Consumption speed histories for $W=(17.5,20,30,40) \delta_{T}$ (resp. cases A to D) are reported in Fig. 9.

For $W$ below the cutoff value (case A), no DL instability occurs, and the flame remains almost planar (as in Fig. 2c), with a normalized consumption speed close to unity.

As expected, the mean values for consumption speed increases with the channel width (case A to D) because the flame has more space to wrinkle and develop a large area compared to the channel cross-section. 


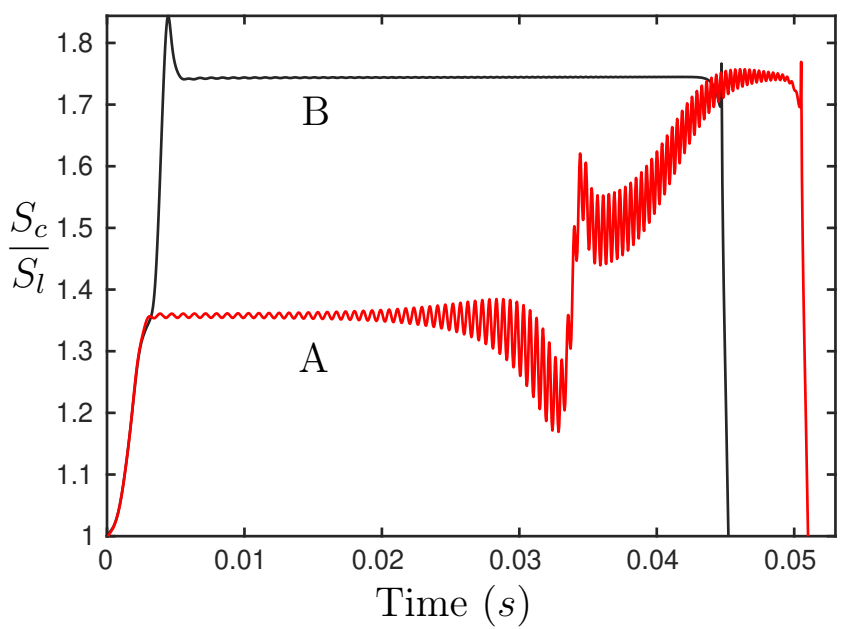

(a)

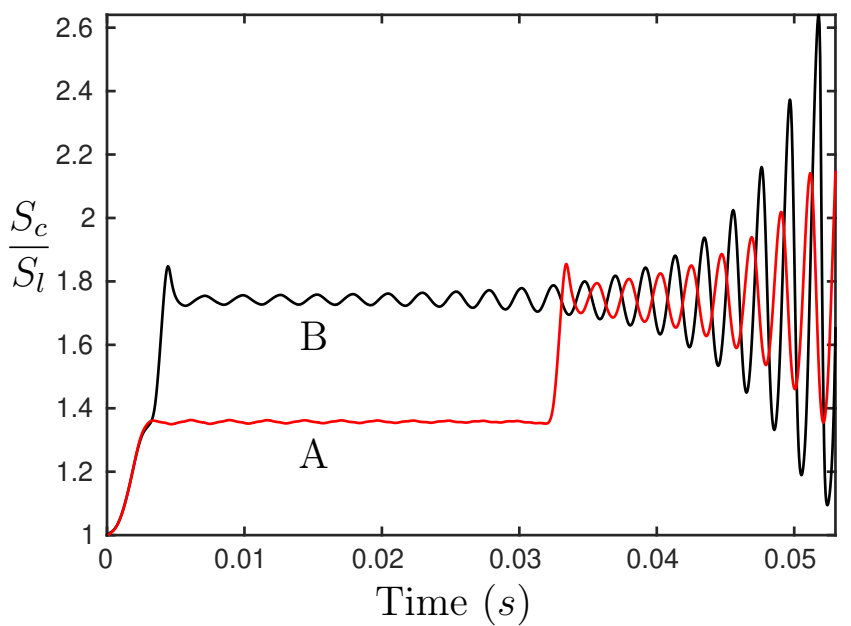

(b)

Figure 8: Flame consumption speed depicting the instabilities in case of initially planar (A) and initially perturbed (B) [Fig. 3] flames, in a channel of width $W=40 \delta_{T}$ : (a) Greater amplitude instabilities seen on symmetric flame (A) for shorter length channel $\left(L=800 \delta_{T}\right) ;(\boldsymbol{b})$ Greater amplitude instabilities seen on non-symmetric flame $(\mathbf{B})$ for longer length channel. $\left(L=2400 \delta_{T}\right)$ 


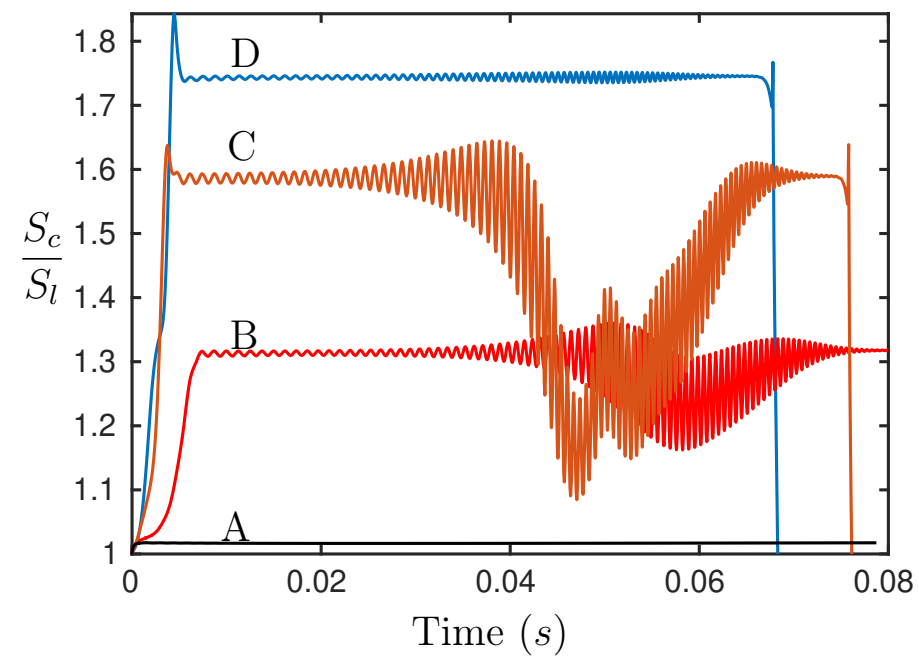

Figure 9: Flame consumption speed in a channel of length $L=1200 \delta_{T}$ and different widths: $(\mathbf{A}): \mathrm{W}=17.5 \delta_{T} ;(\mathbf{B}): \mathrm{W}=20 \delta_{T} ;(\mathbf{C}) \mathrm{W}=30 \delta_{T} ;(\mathbf{D}) \mathrm{W}=40 \delta_{T}$

However, for a channel width over twice the cut-off value (case D), a very small gain was observed compared to intermediate width values (cases $\mathrm{B}$ and $\mathrm{C})$. Interestingly, for these intermediate widths values, one can also observe a significant drop in mean consumption speed, before going back to its steady laminar value. We believe it to be an attempt at flame stabilization by intense thermo-acoustic excitation, a phenomenon to be discussed in the next sub-section.

For further analysis, let us study the flame transfer function, linking the gain of the thermo-acoustic instability with the frequency. The above simulations are repeated in the channel, using non-reflecting conditions, and imposing acoustic fluctuations as in Sec. 4. Asymmetric flames are left to establish for 15ms (see Fig. 10a), time at which the forcing is started. Next, we measure the ratio between the consumption speed amplitude and the 
velocity fluctuation imposed at the inlet (15).

Figure 10a presents the consumption speed histories for two forcing frequencies: $f=1500 \mathrm{~Hz}$ and $f=250 \mathrm{~Hz}$. These eigen frequencies correspond to channel lengths ranging between $(550-1540) \delta_{T}$ for $f=1500 \mathrm{~Hz}$ and $(3300$ $9300) \delta_{T}$ for $f=250 \mathrm{~Hz}$. The flame transfer functions for these two frequencies are reported in Fig. 10b, for above cut-off width $W=(20,30,40,50) \delta_{T}$.

It can be observed that the gain increases with the increase of width at low frequency $(250 \mathrm{~Hz})$ - long channels (as seen in Fig. 10), whereas it decreases with the increase of width at high frequency $(1500 \mathrm{~Hz})$ or shorter channels. This clearly indicates that the channel width has a non-monotonous influence on the instability gain, for varying channel length (or frequency).

In addition, we can infer from Fig. 10b that the gain is lower for high frequencies, a finding in line with the recent study of Jiménez et al. [39]. Note that, for very low frequencies (corresponding to length from $5000 \delta_{T}$ ), it is reported that this tendency is inverted [39].

\subsection{Flames in a long channel}

As a closing section, we shall illustrate the variety of flame propagation behaviors in a longer channel. As most examples above, the width is $W=$ $40 \delta_{T}$, but the length is now $2400 \delta_{T}$, as in Fig. 8.b.

Figure 11.a presents the consumption speed evolution over time in the channel (non-slip boundary conditions), the initially perturbed flame (B) in Fig. 8.b, being a zoom of the same case in the early stages. The second plot of Fig. 11 reports the normalized pressure at the closed end.

Four regions (Z1 to Z4) are identified in the plot, for which successive 


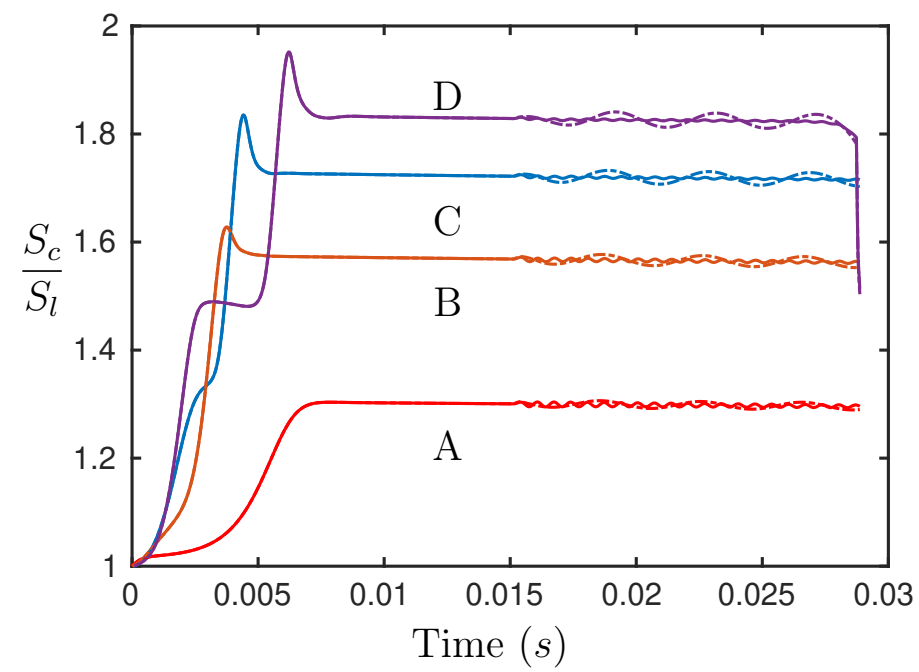

(a)

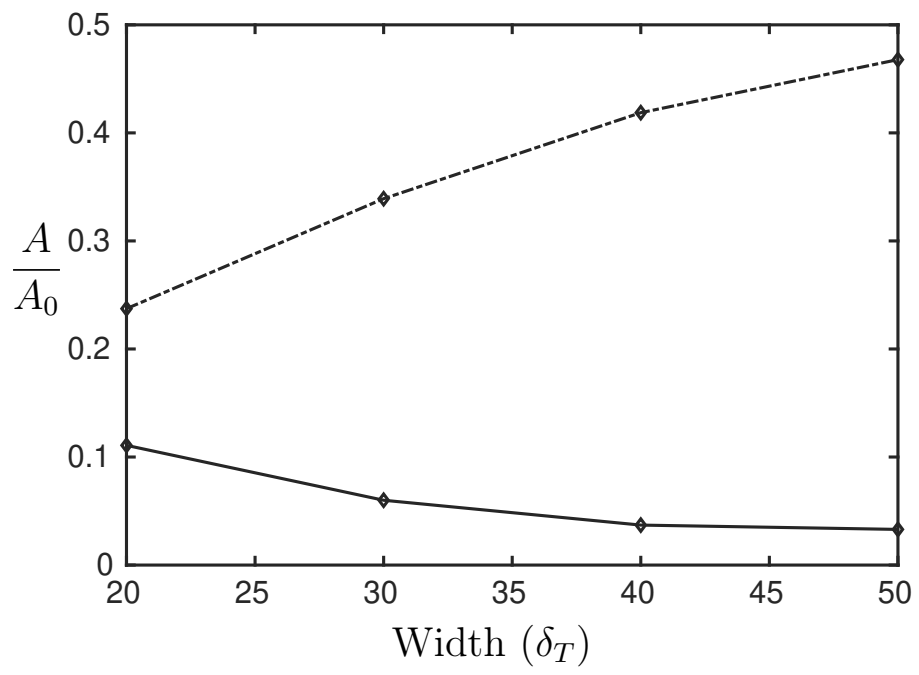

(b)

Figure 10: (a) Acoustic forcing on a flame in different width channels with frequency $f=1500 \mathrm{~Hz}$ in solid and $f=250 \mathrm{~Hz}$ in dash. $\left((\mathbf{A}): \mathrm{W}=20 \delta_{T} ;(\mathbf{B}): \mathrm{W}=30 \delta_{T} ;(\mathbf{C})\right.$ : $\left.\mathrm{W}=40 \delta_{T} ;(\mathbf{D}): \mathrm{W}=50 \delta_{T}\right) ;(\boldsymbol{b})$ Flame Transfer Function $\left(A / A_{0}\right)$ for different widths with frequencies $f=1500 \mathrm{~Hz}$ in solid and $f=250 \mathrm{~Hz}$ in dash. 
flame contours are reported in Fig. 12. We will try, for each zone of Fig. 11, to interpret the evolution of the flame shape coupled to the acoustic forcing.

Let us start with zone Z1. As seen in the first plot of Fig. 12, the amplification of the acoustic pressure seen in this zone corresponds to a nonsymmetric shape for the flame, this shape oscillates at the acoustic period (DL zone of a typical stability diagram such as Fig. 4) leading to acoustics amplification according to the Rayleigh criterion.

We now turn to zone Z2 (second plot of Fig. 12). it can be seen on this plot that after one acoustic period, the flame shape is inverted so that the period of the flame shape is now twice the acoustic period, a typical signature of the parametric instability zone of Fig. 4.

In zone Z3, the acoustic pressure as well as the consumption velocity are now smaller. Figure 12.c where two periods of the acoustic forcing are plotted show that we have now a symmetric shape for the flame (so that the consumption velocity is smaller). The acoustic amplitude has been reduced and the symmetric shape oscillates again at the acoustic period (we are back in a DL zone of a typical stability diagram).

Then the evolution seen in zone Z4 corresponds to a transition of the flame shape to a non-symmetric solution but the flame still oscillates at the acoustic period. Flame profiles are shown in Fig. 12.d, for approximately three acoustic periods.

Having failed to observe a parametrically re-stabilized flat flame in Fig. 11, we reproduced the above simulation considering slip wall and reported the results in Figs. 13 and 14 (with now only two zones identified: Z1, Z2). 
There are some differences between Fig. 11 (non slip walls) and Fig. 13 (slip walls). First of all, as shown in section 3, the DL instability is more important with non slip walls, leading to a higher consumption velocity even without acoustics. The slip wall case is actually more similar to what was obtained in section 4 with periodic boundary conditions, where we observed a perfectly flat parametrically restabilized flame.

The zone Z1 of Fig. 13 is very similar to the case of non-slip boundary conditions, a non-symmetric shape oscillating at the acoustic period, but the situation for zone Z2 is very different, the shape corresponds now to a parametrically flat flame. A fast evolution towards a larger flame surface is seen for a time close to $0.15 \mathrm{~s}$.

Comparing Z3 in Fig. 11 and Z2 in Fig. 13, in which only the boundary condition is modified, the authors are left to wonder if parametrically flat flames may exist in such narrow channels with non-slip boundary conditions.

\section{Conclusions and Perspectives}

In this paper we applied, possibly for the first time, Lattice-Boltzmann numerical methods to the problem of thermo-acoustic instability of premixed flames. The classical configuration of flames propagating in tubes was selected, where acoustics is directly coupled to the flame shape. It was possible to show, both for flames submitted to an imposed acoustic field, and for self-induced thermo-acoustic instability, that the numerical method is able to describe correctly the evolution of the flame.

Future works include studying thermo-acoustic instabilities in more realistic configurations. Application to deflagration to detonation transition 


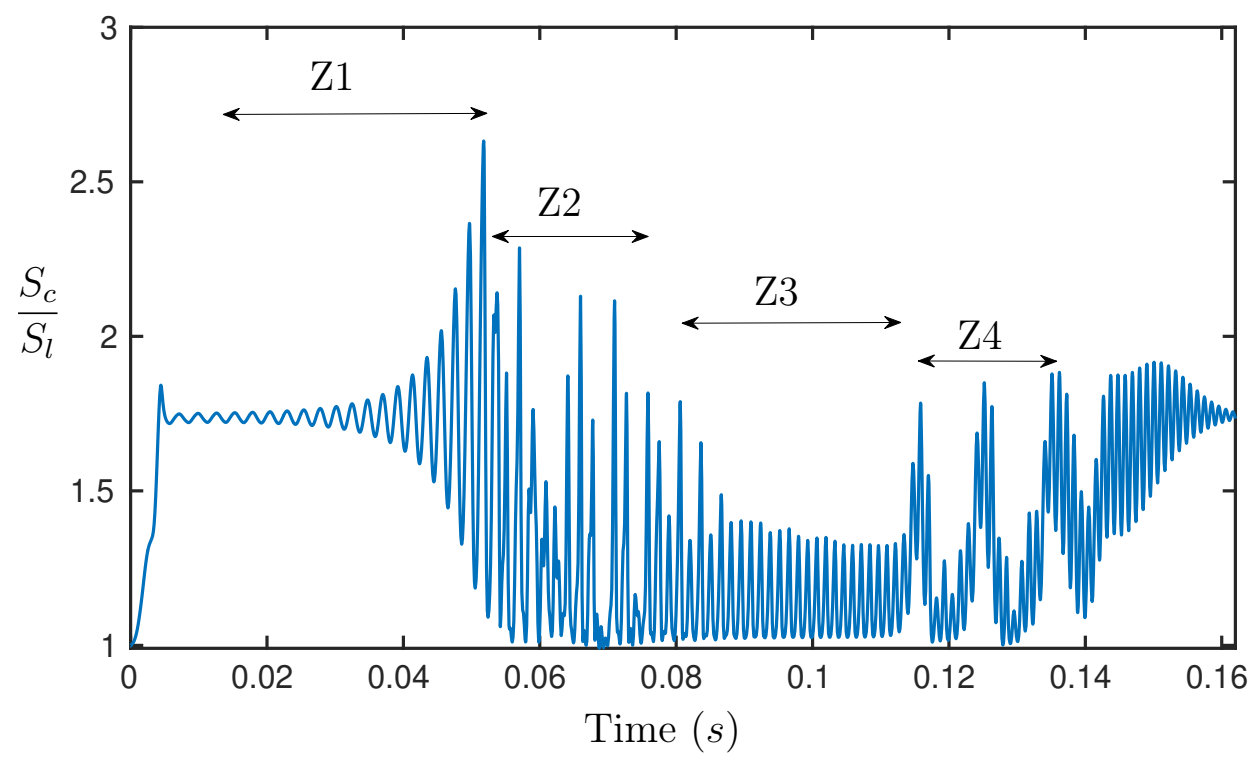

(a)

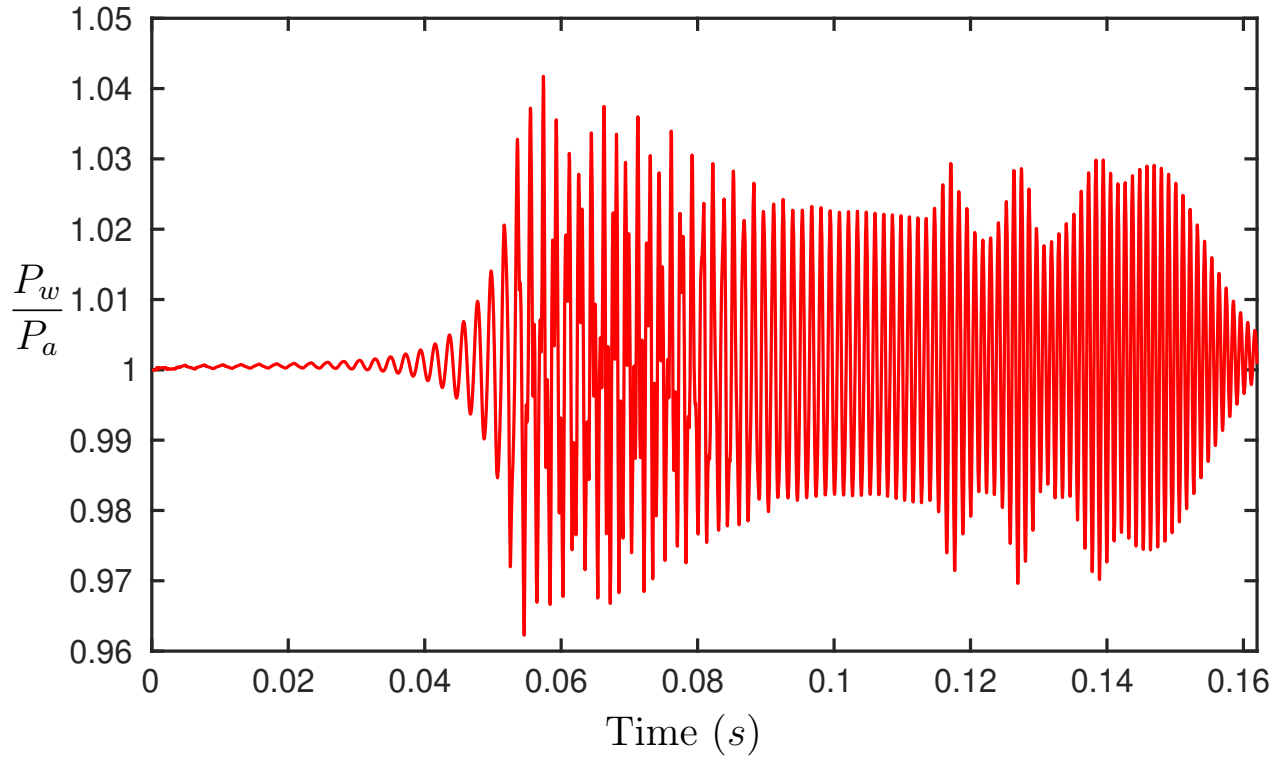

(b)

Figure 11: Thermo-acoustics inside a channel of length $\mathrm{L}=2400 \delta_{T}$ and width $\mathrm{W}=40 \delta_{T}$ : (a) The Flame consumption speed and the different flame regimes observed; (b) Pressure at the closed end wall. 

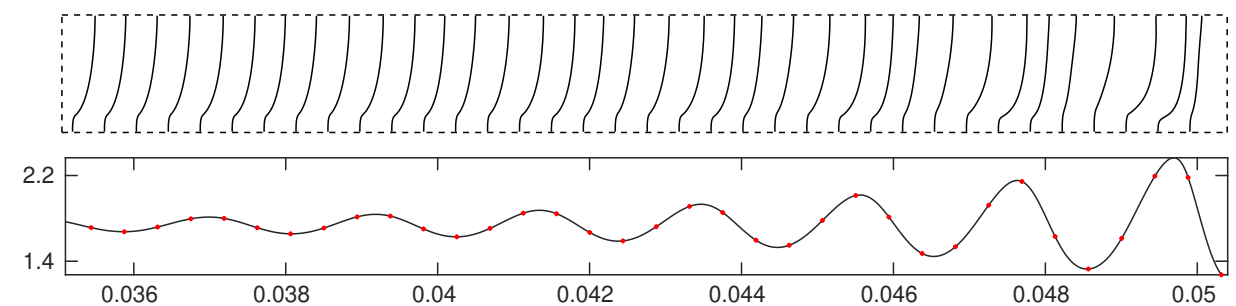

(a)
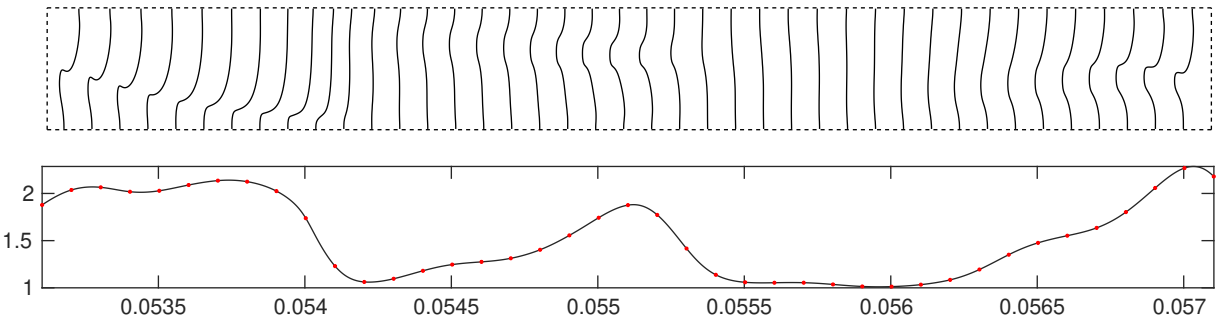

(b)
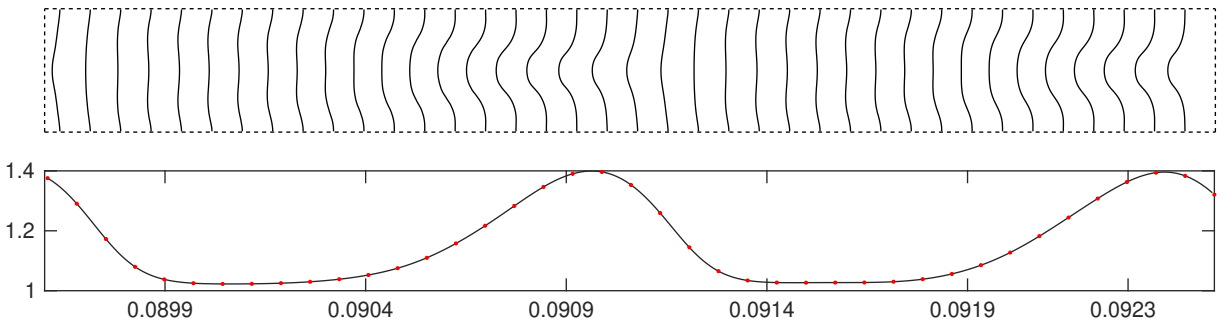

(c)
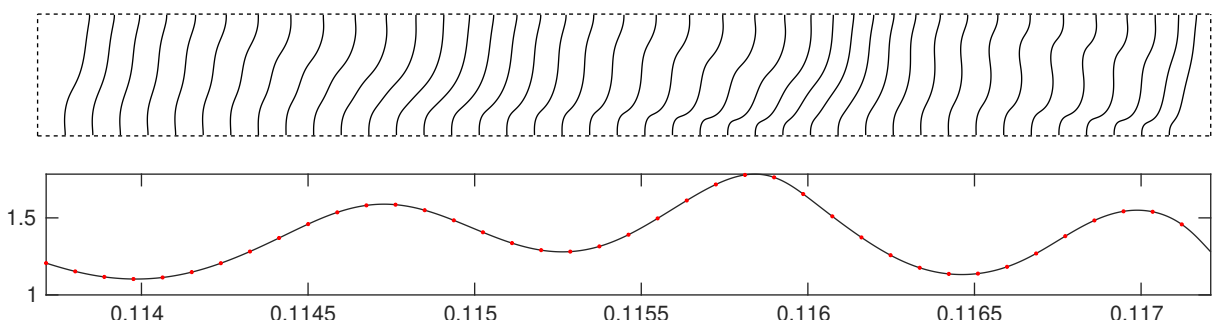

(d)

Figure 12: Iso-contours of temperature representing different regions of instabilities observed in Fig. 11. The graph illustrates the evolution of consumption speed $S_{c}$ with time (in seconds). Red dots represent the time at which the contours are plotted. 


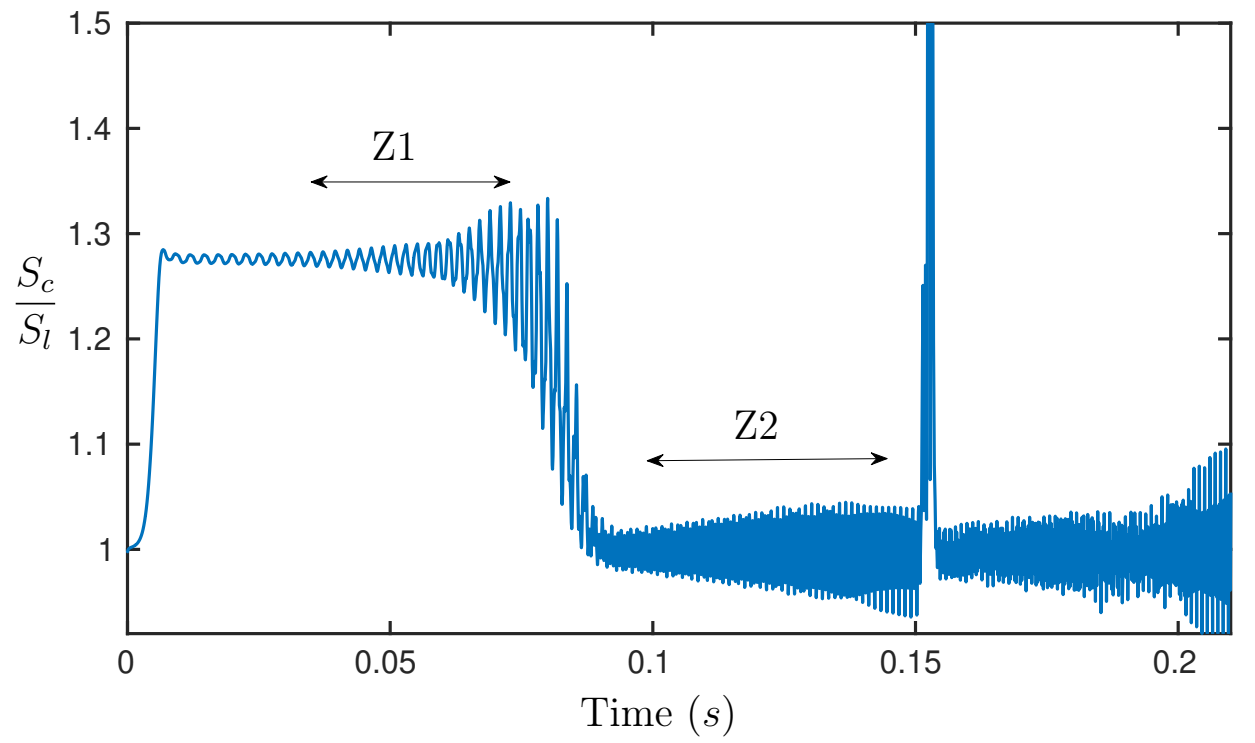

Figure 13: Flame consumption speed inside a slip channel with length $\mathrm{L}=2400 \delta_{T}$ and width $\mathrm{W}=40 \delta_{T}$

will also be investigated.

\section{Acknowledgements}

Carmen Jiménez is gratefully acknowledged for many fruitful discussions, and for providing the results plotted in Fig. C.15. This research was supported by ANR through the MALBEC project (ANR-20-CE05-0009). Centre de Calcul Intensif d'Aix-Marseille and GENCI-TGCC/CINES (Grant 2021-A0092B11951) are acknowledged for granting access to their high performance computing resources. 

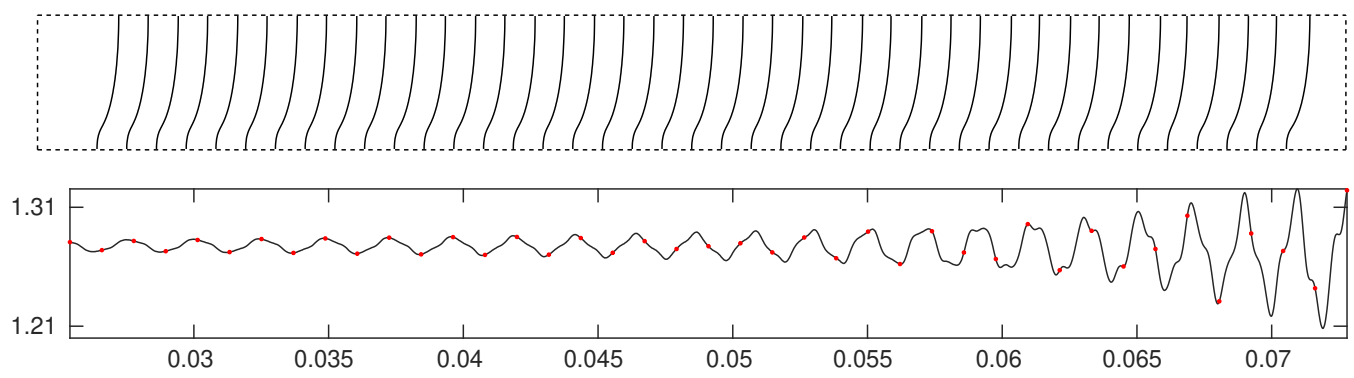

(a)
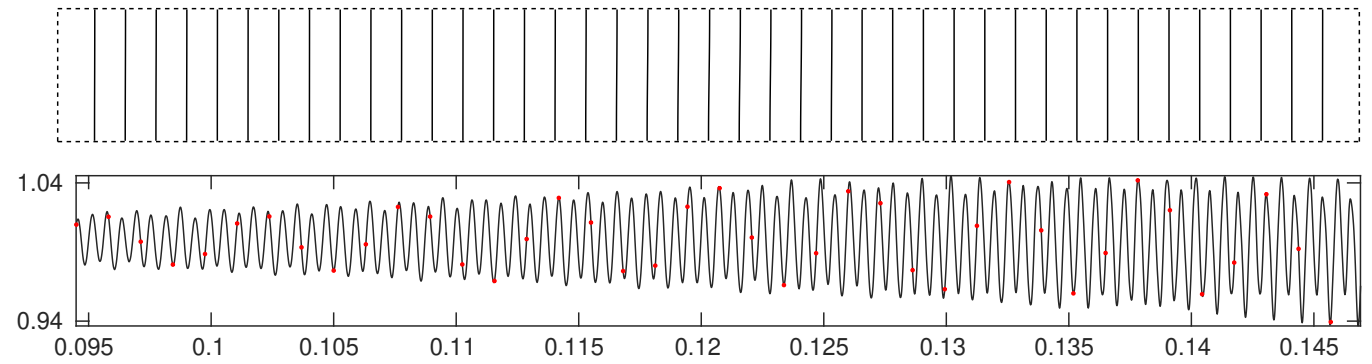

(b)

Figure 14: Iso-contours of temperature representing different regions of instabilities observed in Fig. 13. The graph illustrates the evolution of consumption speed $S_{c}$ with time (in seconds). Red dots represent the time at which the contours are plotted. 


\section{Appendix A. LB method related expressions}

Equilibrium and non-equilibrium functions can be truncated at secondorder as

$$
\begin{aligned}
& f_{i}^{\mathrm{eq}}=w_{i}\left[\rho \theta+\frac{\rho c_{i \alpha} u_{\alpha}}{c_{s}^{2}}+\frac{\mathcal{H}_{i, \alpha \beta}^{(2)} a_{\alpha \beta}^{(2), \mathrm{eq}}}{2 c_{s}^{4}}\right], \\
& f_{i}^{\mathrm{neq}}=\frac{\omega_{i}}{2 c_{s}^{4}} \mathcal{H}_{i, \alpha \beta}^{(2)} a_{\alpha \beta}^{\mathrm{neq}},
\end{aligned}
$$

where,

$$
\begin{aligned}
& \mathcal{H}_{i, \alpha \beta}^{(2)}=c_{i \alpha} c_{i \beta}-c_{s}^{2} \delta_{\alpha \beta}, \quad a_{\alpha \beta}^{(2), \text { eq }}=\rho u_{\alpha} u_{\beta}, \\
& a_{\alpha \beta}^{\text {neq }}=\mathcal{H}_{i, \alpha \beta}^{(2)}\left(f_{i}-f_{i}^{\text {eq }}\right),
\end{aligned}
$$

$\theta$ is the normalized temperature linked with pressure as $p=\rho c_{s}^{2} \theta$, using ideal gas law, $w_{i}$ is the weight coefficient related to the discrete velocity $\boldsymbol{c}_{i}$ and $c_{s}$ is the lattice sound speed. We use the regular D3Q19 lattice, the $\boldsymbol{c}_{i}$ and $w_{i}$ of which can be easily found in the LBM literature e.g. [3].

The reconstruction of macroscopic variables reads

$$
\begin{aligned}
& \rho(t+\Delta t, \boldsymbol{x})=\sum f_{i}^{\mathrm{col}}+\rho(t, \boldsymbol{x})(1-\theta(t, \boldsymbol{x})) \\
& \left(\rho u_{\alpha}\right)(t+\Delta t, \boldsymbol{x})=\sum c_{i \alpha} f_{i}^{\mathrm{col}} .
\end{aligned}
$$

Finally, the required forcing term is obtained as

$$
\begin{aligned}
F_{i}^{E}= & \frac{\omega_{i}}{2 c_{s}^{4}} \mathcal{H}_{i, \alpha \beta}^{(2)}\left[c_{s}^{2} u_{\alpha} \rho_{, \beta}+c_{s}^{2} u_{\beta} \rho_{, \alpha}+\delta_{\alpha \beta} \rho c_{s}^{2}\left(\frac{2}{3}-\frac{\eta_{B}}{\mu}\right) u_{\gamma, \gamma}\right. \\
& \left.+\Delta\left(\rho u_{\alpha} u_{\beta}\right)-\delta_{\alpha \beta} c_{s}^{2} \Delta[\rho(1-\theta)]\right],
\end{aligned}
$$

with $\eta_{B}$ is the bulk viscosity, and

$$
\begin{aligned}
& \Delta\left(\rho u_{\alpha} u_{\beta}\right)=\left(\rho u_{\alpha} u_{\beta}\right)(t+\Delta t, \boldsymbol{x})-\left(\rho u_{\alpha} u_{\beta}\right)(t, \boldsymbol{x}), \\
& \Delta[\rho(1-\theta)]=\rho(t+\Delta t, \boldsymbol{x})(1-\theta(t+\Delta t, \boldsymbol{x}))-\rho(t, \boldsymbol{x})(1-\theta(t, \boldsymbol{x})) .
\end{aligned}
$$




\section{Appendix B. Simulation parameters}

\begin{tabular}{|c|c|c|}
\hline Name & Symbol & Value \\
\hline Mach number & $M a$ & 0.0028 \\
\hline Flame speed & $S_{l}$ & $1 \mathrm{~m} \mathrm{~s}^{-1}$ \\
\hline Activation energy & $E_{a}$ & $30 \mathrm{kcal} \mathrm{mol}^{-1}$ \\
\hline Pre-exponential factor & $A$ & $14.85 \times 10^{14} \mathrm{~cm}^{3} \mathrm{~mol}^{-1} \mathrm{~s}^{-1}$ \\
\hline Thermal diffusivity in unburnt state & $D_{t h}$ & $1 \times 10^{-4} \mathrm{~m}^{2} \mathrm{~s}^{-1}$ \\
\hline Flame diffusive thickness & $\delta_{T}=\frac{D_{t h}}{S_{l}}$ & $1 \times 10^{-4} \mathrm{~m}$ \\
\hline Constant pressure specific heat & $C_{p}$ & $1250 \mathrm{~J} \mathrm{~kg}^{-1} \mathrm{~K}^{-1}$ \\
\hline Thermal expansion parameter & $E_{T}=\frac{T_{b}}{T_{u}}$ & 8 \\
\hline Zeldovich number & $\beta=\frac{E_{a}\left(T_{b}-T_{u}\right)}{R T_{b}^{2}}$ & 5.5 \\
\hline Lewis number & $L e$ & 1 \\
\hline Prandtl number & $\operatorname{Pr}=\frac{\nu}{D_{t h}}$ & 0.15 \\
\hline Acoustic Reynolds number & $R e_{a}=\frac{\delta_{T} \cdot c_{0}}{\nu_{0}}$ & 2225 \\
\hline Space discretization step & $\Delta x$ & $5 \times 10^{-5} \mathrm{~m}$ \\
\hline Time discretization step & $\Delta t$ & $2.624 \times 10^{-8} \mathrm{~s}$ \\
\hline
\end{tabular}

Table B.1: Parameters used in Sec. 3 and Sec. 5 


\begin{tabular}{|c|c|c|}
\hline Name & Symbol & Value \\
\hline \hline Flame speed & $S_{l}$ & $0.20 \mathrm{~m} \mathrm{~s}^{-1}$ \\
\hline Activation energy & $E_{a}$ & $30 \mathrm{kcal} \mathrm{mol}^{-1}$ \\
\hline Pre-exponential factor & $A$ & $4.45 \times 10^{13} \mathrm{~cm}^{3} \mathrm{~mol}^{-1} \mathrm{~s}^{-1}$ \\
\hline Thermal diffusivity in unburnt state & $D_{t h}$ & $2.17 \times 10^{-5} \mathrm{~m}^{2} \mathrm{~s}^{-1}$ \\
\hline Flame diffusive thickness & $\delta_{T}=\frac{D_{t h}}{S_{l}}$ & $1.085 \times 10^{-4} \mathrm{~m}$ \\
\hline Flame transit time & $\tau=\frac{D_{t h}}{S_{l}^{2}}$ & $5.425 \times 10^{-4} \mathrm{~s}$ \\
\hline Expansion parameter & $E_{\rho}=\frac{\rho_{u}}{\rho_{b}}$ & 7.7 \\
\hline Reduced acoustic frequency & $\tilde{\omega_{a}}=2 \pi f \tau$ & 3.40 \\
\hline Inlet perturbation amplitude & $A_{0}$ & $7.5 \times 10^{-4}, 2.5 \times 10^{-3}, 5.5 \times 10^{-3}$ \\
\hline Wave number of structures on the flame & $k=\frac{2 \pi \delta_{T}}{\lambda}$ & 0.08 \\
\hline Prandtl Number & $P r$ & 0.68 \\
\hline Markstein Number & $\mathcal{M}$ & 4.5 \\
\hline Space discretization step & $\Delta x$ & $5 \times 10^{-5} \mathrm{~m}$ \\
\hline Time discretization step & $\Delta t$ & $2.624 \times 10^{-8} \mathrm{~s}$ \\
\hline
\end{tabular}

Table B.2: Parameters used in Sec. 4 


\begin{tabular}{|c|c|c|c|}
\hline Name & Unburnt & Burnt & $S c_{k}$ \\
\hline \hline $\mathrm{C}_{3} \mathrm{H}_{8}$ & 0.0569794 & 0 & 1.241 \\
\hline $\mathrm{O}_{2}$ & 0.260046 & 0.0533094 & 0.728 \\
\hline $\mathrm{CO}_{2}$ & 0 & 0.170602 & 0.941 \\
\hline $\mathrm{H}_{2} \mathrm{O}$ & 0 & 0.0931139 & 0.537 \\
\hline $\mathrm{N}_{2}$ & 0.682975 & 0.682975 & 0.690 \\
\hline
\end{tabular}

Table B.3: Mass fraction composition of fresh and burnt gases composition used in Sec. 3, 4 and 5, and the corresponding Schmidt numbers $S c_{k}$ (used only in Sec. 4: unity Lewis number is used elsewhere). 


\section{Appendix C. Code comparison with the reference paper [39]}

To further validate the LB approach, the model from Jiménez et al. [39], with a single mass fraction equation, was also implemented. Comparison for the pressure evolution at the end wall is provided in Fig. C.15, as obtained with both flow solvers. The tested configuration corresponds exactly to the top plot of Fig. 10.b in [39]. From the results, we can see that the initial

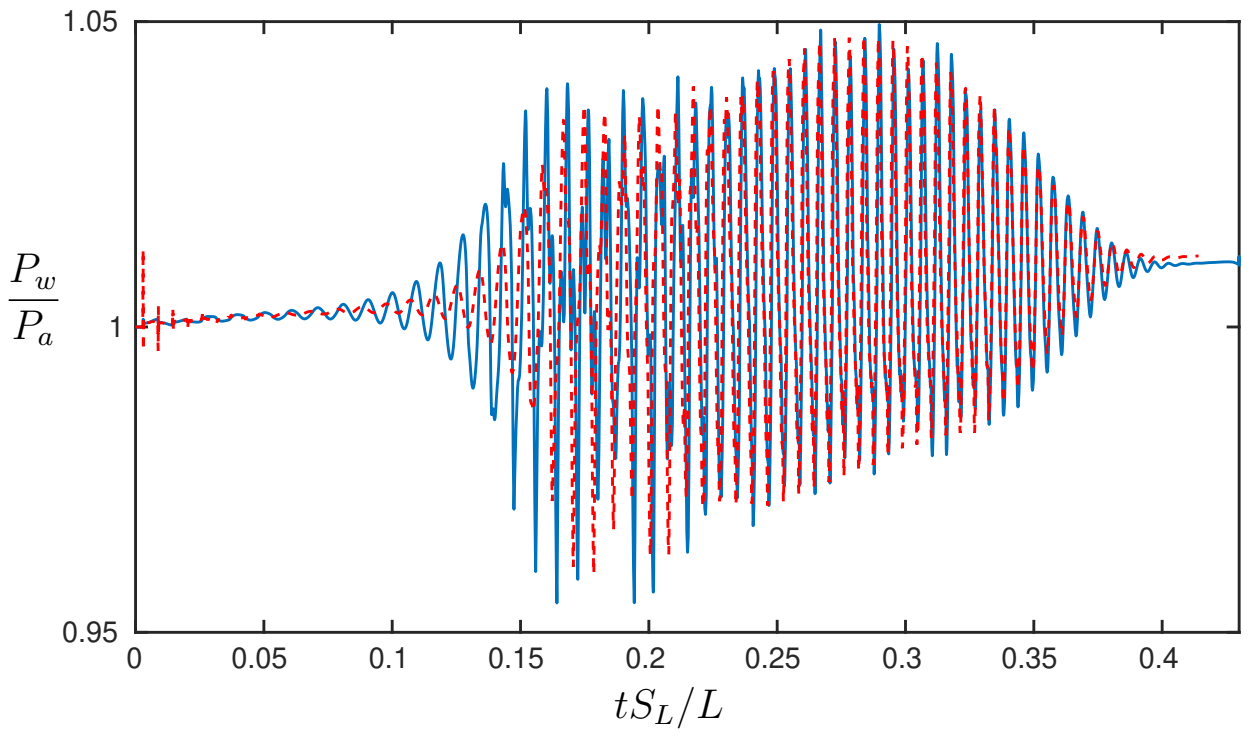

Figure C.15: Pressure at the closed end wall of a channel of $\mathrm{L}=2400 \delta_{T}$ and width $\mathrm{W}=$ $40 \delta_{T}$ : Blue - ProLB ; Red (dashed) - [39]

stages (up to $t S_{L} / L \approx 0.2$ ) differ, most likely due to a different initialization despite our best efforts. Nonetheless, once the flame is well into the tube, an excellent agreement is obtained, both in terms of frequency and amplitude. 


\section{References}

[1] S. Chen, G. D. Doolen, Lattice Boltzmann method for fluid flows, Annu. Rev. Fluid Mech. 30 (1998) 329-364.

[2] A. Sengissen, J.-C. Giret, C. Coreixas, J.-F. Boussuge, Simulations of lagoon landing-gear noise using Lattice Boltzmann solver, in: 21st AIAA/CEAS Aeroacoustics Conference, 2015, p. 2993.

[3] T. Krüger, H. Kusumaatmaja, A. Kuzmin, O. Shardt, G. Silva, E. M. Viggen, The Lattice Boltzmann Method: Principles and Practice, Springer, 2016.

[4] S. Marié, D. Ricot, P. Sagaut, Comparison between Lattice Boltzmann method and navier-stokes high order schemes for computational aeroacoustics, J. Comput. Phys. 228 (2009) 1056-1070.

[5] R. Löhner, Towards overcoming the LES crisis, Int. J. Comput. Fluid Dyn. (2019) 1-11.

[6] S. Succi, G. Bella, F. Papetti, Lattice kinetic theory for numerical combustion, Journal of Scientific Computing 12 (4) (1997) 395-408.

[7] O. Filippova, D. Hänel, A Novel Lattice BGK Approach for Low Mach Number Combustion, Journal of Computational Physics 158 (2) (2000) $139-160$.

[8] K. Yamamoto, X. He, G. Doolen, Simulation of combustion field with lattice Boltzmann method, Journal of statistical physics 107 (April) (2002) 367. 
[9] K. Yamamoto, Combustion Simulation using the Lattice Boltzmann Method, JSME International Journal 47 (2) (2004) 4003 - 409.

[10] K. Yamamoto, N. Takada, LB simulation on soot combustion in porous media, Physica A: Statistical Mechanics and its Applications 362 (1) (2006) 111-117.

[11] S. Guo, Y. Feng, P. Sagaut, Improved standard thermal Lattice Boltzmann model with hybrid recursive regularization for compressible laminar and turbulent flows, Phys. Fluids 32 (2020) 126108.

[12] M. Saadat, S. Hosseini, B. Dorschner, I. Karlin, Extended Lattice Boltzmann model for gas dynamics, Phys. Fluids 33 (2021) 046104.

[13] T. Hanada, T. Kataoka, Lattice Boltzmann method for compressible euler equations based on exact kinetic system, Int. J. Numer. Methods Fluids (2021) 1-16.

[14] J. Latt, C. Coreixas, J. Beny, A. Parmigiani, Efficient supersonic flow simulations using Lattice Boltzmann methods based on numerical equilibria, Philos. Trans. Royal Soc. A 378 (2020) 20190559.

[15] Y. Feng, P. Boivin, J. Jacob, P. Sagaut, Hybrid recursive regularized thermal Lattice Boltzmann model for high subsonic compressible flows, J. Comput. Phys. 394 (2019) $82-99$.

[16] G. Farag, S. Zhao, T. Coratger, P. Boivin, G. Chiavassa, P. Sagaut, A pressure-based regularized Lattice-Boltzmann method for the simulation of compressible flows, Phys. Fluids 32 (2020) 066106. 
[17] S. Guo, Y. Feng, J. Jacob, F. Renard, P. Sagaut, An efficient Lattice Boltzmann method for compressible aerodynamics on d3q19 lattice, J. Comput. Phys. (2020) 109570.

[18] Y. Feng, M. Tayyab, P. Boivin, A Lattice-Boltzmann model for lowmach reactive flows, Combust. Flame 196 (2018) 249 - 254.

[19] M. Tayyab, S. Zhao, Y. Feng, P. Boivin, Hybrid regularized LatticeBoltzmann modelling of premixed and non-premixed combustion processes, Combust. Flame 211 (2020) 173-184.

[20] M. Tayyab, S. Zhao, P. Boivin, Lattice-Boltzmann modelling of a turbulent bluff-body stabilized flame, Phys. Fluids 33 (2021) 031701.

[21] A. Abdelsamie, G. Lartigue, C. E. Frouzakis, D. Thévenin, The TaylorGreen vortex as a benchmark for high-fidelity combustion simulations using low-mach solvers, Comput. Fluids 223 (2021) 104935.

[22] P. Boivin, M. Tayyab, S. Zhao, Benchmarking a Lattice-Boltzmann solver for reactive flows: Is the method worth the effort for combustion?, Phys. Fluids 33 (2021) 017703.

[23] A.-G. Xu, G.-C. Zhang, Y.-B. Gan, F. Chen, X.-J. Yu, Lattice boltzmann modeling and simulation of compressible flows, Frontiers of Physics 7 (5) (2012) 582-600.

[24] B. Yan, A.-G. Xu, G.-C. Zhang, Y.-J. Ying, H. Li, Lattice boltzmann model for combustion and detonation, Frontiers of Physics 8 (1) (2013) 94-110. 
[25] Y.-D. Zhang, A.-G. Xu, J.-J. Qiu, H.-T. Wei, Z.-H. Wei, Kinetic modeling of multiphase flow based on simplified enskog equation, Frontiers of Physics 15 (6) (2020) 1-13.

[26] C. Lin, K. H. Luo, A. Xu, Y. Gan, H. Lai, Multiple-relaxation-time discrete boltzmann modeling of multicomponent mixture with nonequilibrium effects, Physical Review E 103 (1) (2021) 013305.

[27] C. Lin, K. H. Luo, Mesoscopic simulation of nonequilibrium detonation with discrete Boltzmann method, Combustion and Flame 198 (2018) $356-362$.

[28] C. Lin, K. H. Luo, Discrete Boltzmann modeling of unsteady reactive flows with nonequilibrium effects, Physical Review E 99 (1) (2019) 012142 .

[29] D. Casalino, A. F. Ribeiro, E. Fares, S. Nölting, Lattice-Boltzmann aeroacoustic analysis of the lagoon landing-gear configuration, AIAA J. 52 (2014) 1232-1248.

[30] M. R. Khorrami, E. Fares, Simulation-based airframe noise prediction of a full-scale, full aircraft, in: 22nd AIAA/CEAS aeroacoustics conference, 2016, p. 2706.

[31] T. Schuller, T. Poinsot, S. Candel, Dynamics and control of premixed combustion systems based on flame transfer and describing functions, J. Fluid Mech. 894 (2020).

[32] G. H. Markstein, Interaction of Flow Pulsations and Flame Propagation, J. Aeronaut. Sci. 18 (1951) 428-429. 
[33] G. Searby, Acoustic instability in premixed flames, Combust. Sci. Technol. 81 (1992) 221-231.

[34] A. K. Dubey, Y. Koyama, N. Hashimoto, O. Fujita, Experimental and theoretical study of secondary acoustic instability of downward propagating flames: Higher modes and growth rates, Combust. Flame 205 (2019) 316-326.

[35] M. Tayyab, B. Radisson, C. Almarcha, B. Denet, P. Boivin, Experimental and numerical Lattice-Boltzmann investigation of the DarrieusLandau instability, Combust. Flame 221 (2020) 103-109.

[36] G. Searby, D. Rochwerger, A parametric acoustic instability in premixed flames, J. Fluid Mech. 231 (1991) 529-543.

[37] B. Radisson, J. Piketty-Moine, C. Almarcha, Coupling of vibro-acoustic waves with premixed flame, Phys. Rev. Fluids 4 (2019) 121201.

[38] B. Radisson, B. Denet, C. Almarcha, Forcing of a flame by a periodic flow in a Hele-Shaw burner, submitted to Phys. Rev. Fluids (2021).

[39] C. Jiménez, D. Fernández-Galisteo, V. N. Kurdyumov, Flame-acoustics interaction for symmetric and non-symmetric flames propagating in a narrow duct from an open to a closed end, Combust. Flame 225 (2021) 499-512.

[40] A. Petchenko, V. Bychkov, V. Akkerman, L.-E. Eriksson, Flame-sound interaction in tubes with nonslip walls, Combust. Flame 149 (2007) 418434. 
[41] J. Jacob, O. Malaspinas, P. Sagaut, A new hybrid recursive regularised bhatnagar-gross-krook collision model for Lattice Boltzmann methodbased large eddy simulation, J. Turbul. (2018) 1-26.

[42] G. Farag, S. Zhao, G. Chiavassa, P. Boivin, Consistency study of LatticeBoltzmann schemes macroscopic limit, Phys. Fluids 33 (2021) 031701.

[43] T. Poinsot, D. Veynante, Theoretical and numerical combustion, RT Edwards, Inc., 2005.

[44] S. Zhao, G. Farag, P. Boivin, P. Sagaut, Toward fully conservative hybrid lattice boltzmann methods for compressible flows, Physics of Fluids $32(12)(2020) 126118$.

[45] G. Farag, T. Coratger, G. Wissocq, S. Zhao, P. Boivin, P. Sagaut, A unified hybrid lattice-boltzmann method for compressible flows: bridging between pressure-based and density-based methods, Physics of Fluids 33 (8) (2021).

[46] S. Chapman, T. G. Cowling, The Mathematical Theory of Non-Uniform Gases, Cambridge University Press, 1970.

[47] A. Dejoan, C. Jiménez, V. N. Kurdyumov, Critical conditions for nonsymmetric flame propagation in narrow channels: Influence of the flow rate, the thermal expansion, the lewis number and heat-losses, Combust. Flame 209 (2019) 430-440.

[48] B.-T. Chu, L. S. Kovásznay, Non-linear interactions in a viscous heatconducting compressible gas, Journal of Fluid Mechanics 3 (5) (1958) 494-514. 
[49] F. Baillot, D. Durox, S. Ducruix, G. Searby, L. Boyer, Parametric Response of a Conical Flame to Acoustic Waves, Combust. Sci. Technol. 142 (1999) 91-109.

[50] V. V. Bychkov, Analytical scalings for flame interaction with sound waves, Phys. Fluids 11 (1999) 3168-3173.

[51] J. Yáñez, M. Kuznetsov, R. Redlinger, The acoustic-parametric instability for hydrogen-air mixtures, Combust. Flame 160 (2013) 2009-2016.

[52] P. Clavin, P. Garcia, The influence of the temperature dependence of diffusivities on the dynamics, J. de Mec. Theor. et Appl. 2 (1983) 245263.

[53] N. W. McLachlan, Theory and application of Mathieu functions, Publisher to the University Geoffrey Cumberlege, Oxford University Press (1951).

[54] B. Radisson, Dynamique non linéaire de fronts de flammes: expériences et modélisation, Ph.D. thesis, Aix-Marseille (2019).

[55] B. Denet, A. Toma, Numerical Study of Premixed Flames Parametric Acoustic Instability, Combust. Sci. Technol. 109 (1995) 23-33.

[56] O. Colin, F. Ducros, D. Veynante, T. Poinsot, A thickened flame model for large eddy simulations of turbulent premixed combustion, Physics of Fluids 12 (2000). 